\title{
Estimating the phase in ground-based interferometry: performance comparison between single-mode and multimode schemes
}

\author{
E. Tatulli, N. Blind, J.P. Berger, A. Chelli, and F. Malbet
}

\author{
Laboratoire d'Astrophysique, Observatoire de Grenoble, 38041 Grenoble cedex France \\ e-mail: etatulli@obs.ujf-grenoble.fr
}

Received xxx; accepted xxx

\begin{abstract}
Aims. In this paper we compare the performance of multi and single-mode interferometry for the estimation of the phase of the complex visibility.

Methods. We provide a theoretical description of the interferometric signal which enables to derive the phase error in presence of detector, photon and atmospheric noises, for both multi and single-mode cases.

Results. We show that, despite the loss of flux occurring when injecting the light in the single-mode component (i.e. single-mode fibers, integrated optics), the spatial filtering properties of such single-mode devices often enable higher performance than multimode concepts. In the high flux regime speckle noise dominated, single-mode interferometry is always more efficient, and its performance is significantly better when the correction provided by adaptive optics becomes poor, by a factor of 2 and more when the Strehl ratio is lower than $10 \%$. In low light level cases (detector noise regime), multimode interferometry reaches better performance, yet the gain never exceeds $\sim 20 \%$, which corresponds to the percentage of photon loss due to the injection in the guides. Besides, we demonstrate that single-mode interferometry is also more robust to the turbulence in both cases of fringe tracking and phase referencing, at the exception of narrow field of views $\left(\lesssim 1^{\prime \prime}\right)$.

Conclusions. Our conclusion is therefore that, from a theoretical point of view and contrarily to a widespread opinion, fringe trackers built using single-mode optics should be considered as a solution both practical and competitive.
\end{abstract}

Key words. Instrumentation: interferometers - Methods: analytical - Techniques: interferometric

\section{Introduction}

Performance of ground-based optical interferometers is severely limited by the atmospheric turbulent piston which introduces a random optical path difference between the beams that are combined to produce fringes. These fringes are thus randomly moving on the detector, blurring the signal and preventing to integrate on time longer than the coherence time of the atmosphere, typically a few tens of milliseconds in the infrared. As a consequence, the limitingmagnitude and ultimate precision of ground-based interferometers are dramatically reduced.

By estimating and compensating in real-time the interferometric phase - in other words by "locking" the fringes on the detector, fringe tracking and phase referencing devices are powerful instruments to circumvent this problem, allowing to integrate the interferograms on much longer time frames. Such instruments, which noticeably improve the sensitivity and the accuracy of interferometers are since a few years undergoing major developments, as can attest the amount of concepts which are currently studied for various interferometers (Le Bouquin et al. 2008; Sahlmann et al. 2008. VLTI; Berger et al. 2008 CHARA; Jurgenson et al. 2008. MROI).

In all the different concepts proposed, one key issue still in debate among the instrumental community is the relevance of using spatial filtering devices such as single-mode fibers or integrated optics to carry/combine the beams. It is often argued that spatial filtering is not suited for that type of instruments since only a fraction of the total flux is injected in the spatial filter component. This coupling efficiency, which is typically of the order of the Strehl ratio (Coudé du Foresto et al. 2000), can indeed be low in presence of strong turbulence and/or poor adaptive optics (AO) correction. However, the issue is not that simple. Singlemode filters only keeps the part of the incoming flux which is related to the coherent part of the corrugated wavefront. In other words single-mode devices only propagate the first mode of the electro-magnetic field leaving at its output a plane wavefront - that is a deterministic signal, the price to pay being a loss of flux correlated to the strength of the turbulence. At the contrary, multimode schemes such as bulk optics are preserving the total flux but keeps at the output the incoherent part of the wavefront (producing randomly moving speckle patterns in the image) which makes the interferogram sensitive to turbulence. Hence choosing between single-mode and multimode schemes corresponds to decide whether losing flux or losing coherence in the signal will be the best strategy to optimize the performance of the interferometer.

As a matter of fact, the efficiency of single-mode devices in terms of precision and robustness of the estimation of the amplitude of the visibility has already been demonstrated experimentally (Coudé du Foresto et al. 1997) and theoretically as well (Tatulli et al. 2004). However concerning the estimation of phase - which is the quantity of interest for fringe tracking and phase referencing instruments, the situation is not clear yet. If simulations have been initiated 


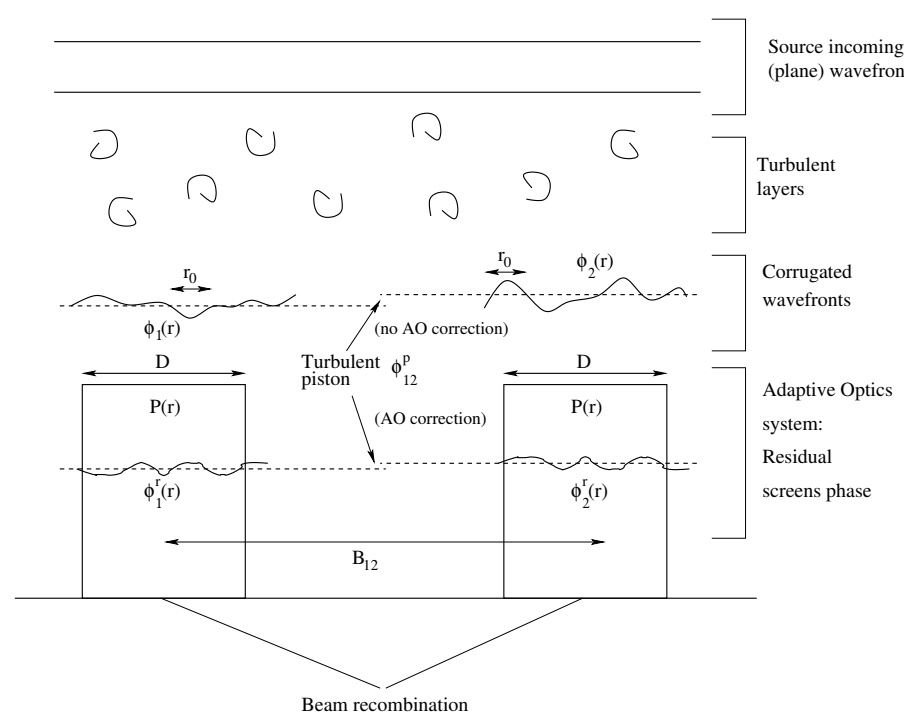

Fig. 1. Sketch of the effect of the turbulence in interferometry. On each telescope pupil $P(\boldsymbol{r})$ separated by the baseline distance $B_{12}$, the wavefront is affected by a random phase screen, respectively $\phi_{1}(\boldsymbol{r})$ and $\phi_{2}(\boldsymbol{r})$. The strength of the turbulence depends on the parameters $D / r_{0}$ where $D$ is the diameter of the telescope and $r_{0}$ is the Fried parameter (Fried 1965), describing the typical size of a coherent cell in the wavefront. Furthermore, the optical path difference between the two beams is randomly shifted by the turbulent piston $\phi_{12}^{p}$ which is the phase difference between the two telescopes, averaged on the pupils. If an AO system is present on each telescope, then the wavefront is partially corrected in real time, and the quantity of interest are the residual phases $\phi_{1}^{r}(\boldsymbol{r})$ and $\phi_{2}^{r}(\boldsymbol{r})$ and the associated residual turbulent piston.

in some specific cases to estimate the effects of spatial filtering (Buscher et al. 2008 phase jumps; Tubbs 2005 computation of the coherence time), no theoretical formalism regarding this issue has, to our knowledge, been presented so far.

In this paper, we derive the error of the interferometric phase in presence of detector, photon and atmospheric noises, both for the single-mode and multimode cases in presence of partial AO correction, following a formalism analogue to the one previously developed for the squared visibility in Tatulli et al. (2004). We then compare the performance of single-mode and multimode schemes for the estimation of the phase of the interferograms, and apply our analysis in the framework of fringe-tracking and phase referencing methods.

\section{The phase in ground-based interferometry}

\subsection{General description and underlying assumptions}

The ground-based interferometer: As sketched in Fig. 1. we consider an interferometer with two telescopes, separated by the baseline distance $B_{12}$. We assume that both telescope are identical, that is they are described by the same pupil function $P(r)$ of diameter $D$, therefore by the same collecting area $\Sigma_{P}$ defined as $\Sigma_{P}=\int[P(\boldsymbol{r})]^{2} \mathrm{~d} \boldsymbol{r}$. Their transmission are however different, namely $t_{1}$ and $t_{2}$ respectively. The wavefront over each telescope is affected by randomly moving phase screens, respectively $\phi_{1}(r)$ and $\phi_{2}(r)$. The typical cell size of these turbulent phases is $r_{0}$ (Fried 1965) and the strength of the turbulence is characterized by the quantity $D / r_{0}$. When Adaptive Optic systems are present on each telescope, they partially compensate in real-time the wavefront corrugations of the atmosphere. The correction is however not perfect and it remains residual phases $\phi_{1}^{r}(r)$ and $\phi_{2}^{r}(r)$ affecting the two telescopes. Because of this turbulence, the optical path difference (opd) between the two light paths to the star through the two telescopes is randomly shifted by the so-called turbulent piston $\phi_{12}^{p}$, i.e. the phase difference between the two telescopes averaged over the pupils:

$$
\phi_{12}^{p}=\int P(\boldsymbol{r}) \phi_{1}^{r}(\boldsymbol{r}) \mathrm{d} \boldsymbol{r}-\int P(\boldsymbol{r}) \phi_{2}^{r}(\boldsymbol{r}) \mathrm{d} \boldsymbol{r}
$$

The interferogram: A 2-telescope interferogram consists in an incoherent part, which is the sum of the photometric fluxes coming from both telescopes, and a modulated coherent part - namely the fringes, proportional to the socalled complex coherent flux $F_{12}^{c}$ which depends on the flux $N$ (photons per surface unit and per time unit) and on the complex visibility $V_{12}$ of the source corresponding to the baseline frequency $f_{12}=B_{12} / \lambda$ of the interferometer. $\lambda$ is the effective wavelength of the interferogram. The modulation of the fringes can be whether temporal when the opd is scanned with moving piezo-mirrors (temporal coding), whether spatial when the opd is scanned with dedicated output pupils which separation defines the frequency of the modulation (spatial coding).

Finally, the phase of the interferogram $\Phi_{12}$, that is the phase shift with respect to the zero opd, originates from the source phase $\phi_{12}^{o b j}$, a potential instrumental phase $\phi_{12}^{i n s}$, that we will assume equal to zero in the following ${ }^{1}$, and the turbulence piston phase $\phi_{12}^{p}$.

\subsection{Estimating the interferometric phase and its associated error}

Estimating the interferometric phase $\Phi_{12}$ requires to define and compute from each interferogram an appropriate complex estimator $\widetilde{F_{12}^{c}}$ of the complex coherent flux $F_{12}^{c}$. Several methods are possible to build this estimator such as "ABCD" techniques in the image plane (Colavita 1999. Tatulli et al. 2007) or analysis in the Fourier plane (Roddier \& Lena 1984: Coudé du Foresto et al. 1997). In any case, the measured phase is then the argument of the complex estimator $\widehat{F_{12}^{c}}$ :

$$
\Phi_{12}=\operatorname{atan}\left[\frac{\operatorname{Im}\left(\widetilde{F_{12}^{c}}\right)}{\operatorname{Re}\left(\widetilde{F_{12}^{c}}\right)}\right]
$$

In this framework, one can show that in first approximation, i.e. when the error on $\widetilde{F_{12}^{c}}$ is small compared to its amplitude, the variance of the instantaneous phase (i.e. measured over one interferogram) can be expressed as following

\footnotetext{
1 considering a non zero instrumental phase $\phi_{12}^{\text {ins }}$ would only introduce an extra shift of the interferogram but would not affect the performance of the interferometer, as long as this instrumental phase is stable or calibratable.
} 
Table 1. Expression of the variance of the phase for the three noise regimes, respectively detector, photon and atmospheric regimes, both in the multimode and single-mode cases. See text and Appendix $\mathrm{A}$ for a description of the parameters involved.

$$
\begin{aligned}
& \frac{\text { Multimode case }}{\sigma_{\text {det }_{\phi}}^{2}=\frac{1}{2} \frac{N_{\text {pix }} \sigma_{\text {det }}^{2}}{N^{2} t_{1} t_{2}\left|V_{12}\right|^{2} \mathrm{e}^{-2 \sigma_{\phi_{r}}^{2}}}} \\
& \sigma_{p h o t_{\phi}}^{2}=\frac{1}{2} \frac{t_{1}+t_{2}}{N t_{1} t_{2}\left|V_{12}\right|^{2} \mathrm{e}^{-2 \sigma_{\phi_{r}}^{2}}} \\
& \sigma_{a t m_{\phi}}^{2}=\frac{1}{2} \frac{\int\left[P(\boldsymbol{r}) P\left(\boldsymbol{r}^{\prime}\right) \mathrm{e}^{-\frac{1}{2} \mathcal{D}_{\phi_{r}}\left(\boldsymbol{r}, \boldsymbol{r}^{\prime}\right)}\right]^{2} \mathrm{~d} \boldsymbol{r} \mathrm{d} \boldsymbol{r}^{\prime}-\mathrm{e}^{-4 \sigma_{\phi_{r}}^{2}} \int\left[P(\boldsymbol{r}) P\left(\boldsymbol{r}^{\prime}\right) \mathrm{e}^{\frac{1}{2} \mathcal{D}_{\phi_{r}}\left(\boldsymbol{r}, \boldsymbol{r}^{\prime}\right)}\right]^{2} \mathrm{~d} \boldsymbol{r} \mathrm{d} \boldsymbol{r}^{\prime}}{\left[\int P^{2}(\boldsymbol{r}) \mathrm{d} \boldsymbol{r}\right]^{2} \mathrm{e}^{-2 \sigma_{\phi_{r}}^{2}}}
\end{aligned}
$$

Single-mode case

$$
\begin{aligned}
\sigma_{\text {det }_{\phi}}^{2} & =\frac{1}{2} \frac{N_{\text {pix }} \sigma_{\text {det }}^{2}}{\rho_{0}^{2} N^{2} t_{1} t_{2}\left|V_{12}\right|^{2} \mathrm{e}^{-2 \sigma_{\phi_{r}}^{2}}} \\
\sigma_{\text {phot }_{\phi}}^{2} & =\frac{1}{2} \frac{\left(t_{1}+t_{2}\right) \overline{\mathcal{S}}}{\rho_{0} N t_{1} t_{2}\left|V_{12}\right|^{2} \mathrm{e}^{-2 \sigma_{\phi_{r}}^{2}}} \\
\sigma_{\text {atm }_{\phi}}^{2} & =\frac{1}{2} \frac{\left[\int P(\boldsymbol{r}) P\left(\boldsymbol{r}^{\prime}\right) \mathrm{e}^{-\frac{1}{2} \mathcal{D}_{\phi_{r}}\left(\boldsymbol{r}, \boldsymbol{r}^{\prime}\right)} \mathrm{d} \boldsymbol{r} \mathrm{d} \boldsymbol{r}^{\prime}\right]^{2}-\mathrm{e}^{-4 \sigma_{\phi_{r}}^{2}\left[\int P(\boldsymbol{r}) P\left(\boldsymbol{r}^{\prime}\right) \mathrm{e}^{\frac{1}{2} \mathcal{D}_{\phi_{r}}\left(\boldsymbol{r}, \boldsymbol{r}^{\prime}\right)} \mathrm{d} \boldsymbol{r} \mathrm{d} \boldsymbol{r}^{\prime}\right]^{2}}}{\left[\int P(\boldsymbol{r}) \mathrm{d} \boldsymbol{r}\right]^{4} \mathrm{e}^{-2 \sigma_{\phi_{r}}^{2}}}
\end{aligned}
$$

(Chelli 1989, see Eq. (5), case $j=k$ ):

$$
\sigma_{\phi}^{2}=\frac{1}{2} \frac{\mathrm{E}\left(\left|\widetilde{F_{12}^{c}}\right|^{2}\right)-\operatorname{Re}\left[\mathrm{E}\left({\widetilde{F_{12}^{c}}}^{2}\right)\right]}{\left[\mathrm{E}\left(\widetilde{F_{12}^{c}}\right)\right]^{2}}
$$

where E denotes the expected value. Chelli (1989) has also demonstrated that the variance of the interferometric phase is independent of the object phase. For sake of simplicity, we will therefore consider that the source of interest is centrosymmetric, namely $\phi_{12}^{o b j}=0$.

Regardless of the method used to build the estimator from the interferogram, the statistics of the phase will depends on whether the interferometer is using a multimode or a single-mode design. In the following, we explore how the atmospheric spatial fluctuations of the turbulent wavefront affects the fringe pattern, in both multimode and singlemode cases.

\subsection{The coherent flux in multimode interferometry}

In multimode interferometry, the total flux on the detector remains constant (neglecting scintillation) and the interferograms are created in speckle patterns randomly moving with the fluctuations of the turbulent wavefronts over the two telescopes.

There are two different ways to combine the beams in multimode interferometry (Chelli \& Mariotti 1986): whether in the image plane, a technique which is suited to perform spatial coding of the fringes on the detector, as known as Fizeau (Beckers \& Hege 1984) and Michelson (Mourard et al. 1994) mounting: ${ }^{2}$ or in the pupil plane which is commonly used to temporally sample the interferogram through dedicated moving piezo-electric mirrors (Dyck et al. 1995). In both cases however, the expression of the coherent flux can be written as following (as shown in the demonstration of Appendices A.3.1 and A.3.2 of this paper and in Appendices A1 and A2 of Buscher et al. 2008):

$$
F_{12}^{c}=\Sigma_{P} N \sqrt{t_{1} t_{2}} V_{12} T_{12}
$$

$T_{12}$ is the normalized interferometric transfer function resulting of the cross-correlation of the pupil of the two telescopes, corrugated by the residual atmospheric screen phases $\phi_{1}^{r}(\boldsymbol{r})$ and $\phi_{2}^{r}(\boldsymbol{r})$, (see Fig. 1). It writes:

$$
T_{12}=\frac{\int[P(\boldsymbol{r})]^{2} \mathrm{e}^{i\left(\phi_{1}^{r}(\boldsymbol{r})-\phi_{2}^{r}(\boldsymbol{r})\right)} \mathrm{d} \boldsymbol{r}}{\int[P(\boldsymbol{r})]^{2} \mathrm{~d} \boldsymbol{r}}
$$

As an analogy with classical AO systems on single-pupil telescopes (see e.g. Fusco \& Conan 2004), $\left|T_{12}\right|^{2}$ can be seen as the instantaneous interferometric Strehl ratio, in the case of multimode interferometry.

\subsection{The coherent flux in single-mode interferometry}

The main property of single-mode devices such as fibers or integrated optic chips is to perform a spatial filtering of the input wavefront so that only its Gaussian part is transmitted. As a consequence, a single-mode device turns the input spatial wavefront fluctuations into intensity fluctuations at the output. Doing so, each outgoing wavefront is flat, hence

\footnotetext{
2 in Fizeau combination, the output pupil is homothetic to the entrance one, whereas in Michelson scheme this property is not conserved.
} 
E. Tatulli et al.: Multimode vs. single-mode interferometric phase

magnitude
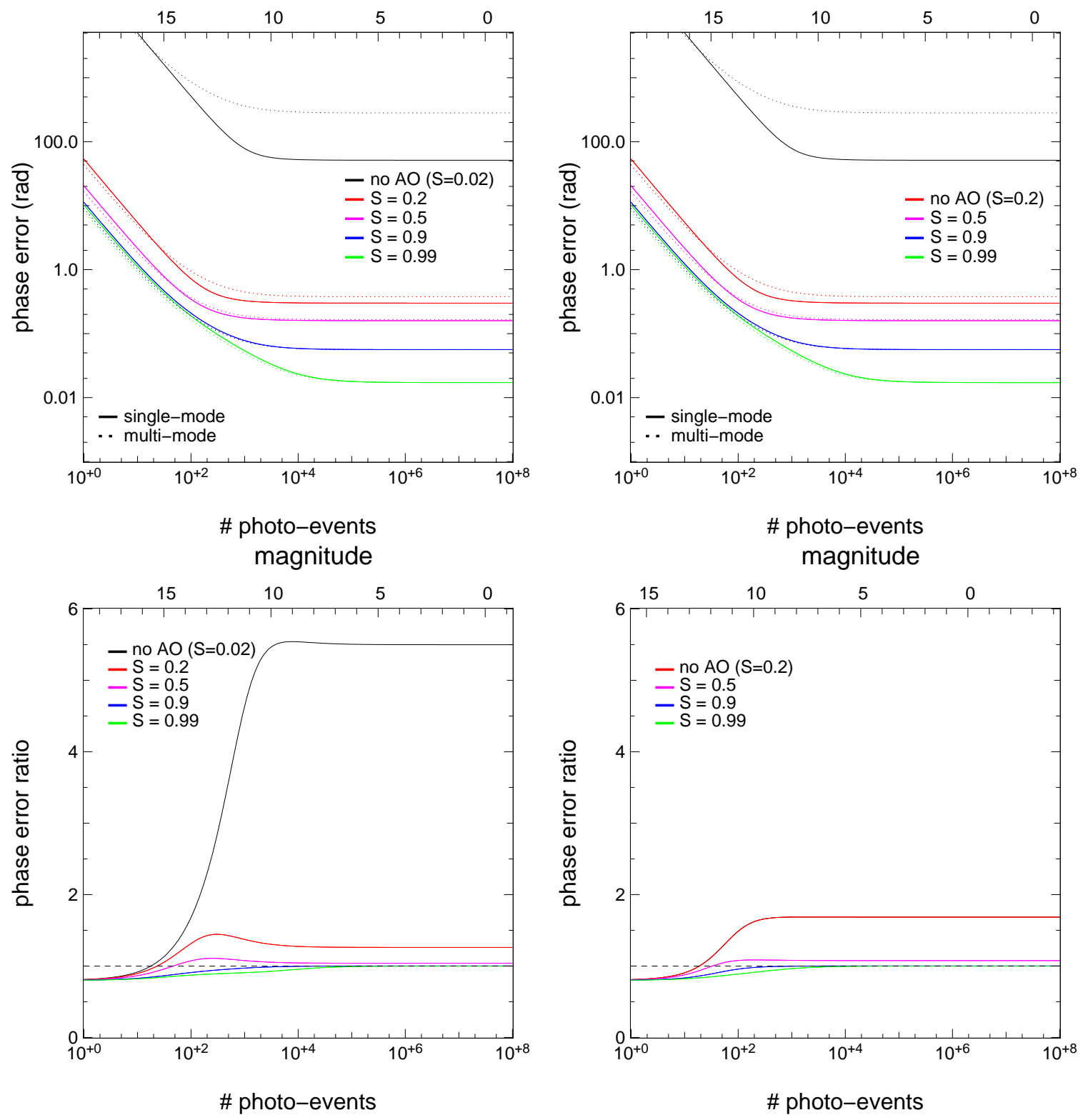

Fig. 2. Global performance comparison between multimode and single-mode interferometry, all sources of noise being considered. Top: Error of the phase in multimode (dotted lines) and single-mode (solid lines) as the function of the number of detected photoevents (per time unit) and K-band magnitude. For both cases, we can see the three regimes: the detector noise (in $1 / N)$, then (shortly) the photon noise (in $1 / \sqrt{N}$ ) and finally the speckle noise (saturation). Bottom: Ratio of multimode vs. single-mode phase error. Plots are shown for several levels of correction (Strehl ratios). The fixed parameters are $\sigma_{\text {det }}=4 e^{-} / \mathrm{pix},|V i j|=1, N_{p i x}=4$. Two turbulence strengths are considered $D / r_{0}=8$ (left) and $D / r_{0}=2$ (right) which correspond to the average turbulence conditions of the VLTI with UTs $(D=8 \mathrm{~m})$ and ATs $(D=1.8 \mathrm{~m})$ respectively. For the conversion between detected photoevents and magnitude scale, we have considered a total transmission coefficient of $15 \%$, a detector quantum efficiency of $50 \%$, an integration time of $30 \mathrm{~ms}$ and a spectral resolution of 35 with 2 pixels by spectral channel.

the shape of the interferogram is deterministic, only depending on the instrumental configuration. The trade-off is that only a fraction of the flux is transmitted, corresponding to the coherent energy of the turbulent wavefront. The single-mode instantaneous coherent flux thus takes the form (see Appendix A.4. Eq. A.38):

$$
F_{12}^{c}=\Sigma_{P} N \sqrt{t_{1} t_{2}} \rho^{12}(V)
$$

where $\rho^{12}(V)$ is the interferometric coupling coefficient that depends both on the source extension and on the level of AO correction (Tatulli et al. 2004, see Eq. (3), and Eq. A.35, Appendix A.4 of this paper). Focusing on compact sources, that is astrophysical objects unresolved by a single telescope, $\rho^{12}(V)$ takes a simple expression of the form $\rho^{12}(V)=\rho_{0} V_{12} \rho_{12}$ (Tatulli \& Chelli 2005, Eqs. $\left.(4,5,6)\right)$. 
The coherent flux thus rewrites:

$$
F_{12}^{c}=\Sigma_{P} N \sqrt{t_{1} t_{2}} \rho_{0} V_{12} \rho_{12}
$$

where $\rho_{12}$ is independent of the source properties:

$$
\rho_{12}=\frac{\int P(\boldsymbol{r}) \mathrm{e}^{i\left(\phi_{1}^{r}(\boldsymbol{r})\right)} \mathrm{d} \boldsymbol{r}}{\int P(\boldsymbol{r}) \mathrm{d} \boldsymbol{r}} \frac{\int P(\boldsymbol{r}) \mathrm{e}^{-i\left(\phi_{2}^{r}(\boldsymbol{r})\right)} \mathrm{d} \boldsymbol{r}}{\int P(\boldsymbol{r}) \mathrm{d} \boldsymbol{r}}
$$

and where $\rho_{0}$ is the maximum achievable coupling efficiency, shown to be $\sim 80 \%$ (Shaklan \& Roddier 1988), due to geometrical mismatch between the telescope Airy disk profile and the Gaussian profile of the propagated mode.

Eq. (8) has to be compared with Eq. (5). We can see that they are almost similar, but with one major difference: in Eq. (5), the product of the phasors is integrated over the pupil whereas in Eq. (8), each phasor is first integrated over the pupil, then the product is performed. As noticed by Buscher et al. (2008), such difference is the mathematical expression of the property of spatial filtering.

Again, $\left|\rho_{12}\right|^{2}$ - which is equal to 1 in the case of perfect $\mathrm{AO}$ correction/absence of atmospheric turbulence - can be seen by as the instantaneous interferometric Strehl ratio, but for the single-mode case.

\section{Performance comparison}

\subsection{Phase noise}

From Eqs. (3, 4.7), we derive the expression of the error of the phase for both single-mode and multimode cases, as developed in Appendix A. We show that the variance of the interferometric phase can be decomposed as the quadratic sum of three terms, corresponding to the three regimes respectively detector, photon and atmospheric regimes:

$$
\sigma_{\phi}^{2}=\sigma_{\operatorname{det}_{\phi}}^{2}+\sigma_{p h o t_{\phi}}^{2}+\sigma_{a t m_{\phi}}^{2}
$$

where the detail of each term is given in Table 1. The phase error depends on parameters coming from the source and the instrument which we recall here: $N$ is the number of incoming photons per surface unit and per time unit, $\left|V_{12}\right|$ is the amplitude of the visibility, $t_{1}$ and $t_{2}$ are the transmissions of both telescopes, $N_{p i x}$ is the number of pixels to sample the interferogram, $\sigma_{\text {det }}$ is the detector noise. The phase error also depends on atmospheric terms: the long exposure Strehl ratio $\overline{\mathcal{S}}$ and its associated coherent energy $\mathrm{e}^{-\sigma_{\phi_{r}}^{2}}$ where $\sigma_{\phi_{r}}^{2}$ is the variance of the residual phase of a single telescope (Noll 1976), and of the phase structure function in presence of partial $\mathrm{AO}$ correction $\mathcal{D}_{\phi_{r}}(\boldsymbol{r})$ as modelled in Appendix D.

Figure 2 (top) shows the multimode and single-mode phase error as a function of the number of incoming photoevents (alternatively, source $K$-band magnitude), for different levels of AO correction (i.e. different Strehl ratios). In a general way, the behavior of the error is in both cases similar: at low fluxes, the detector noise dominates with a slope of the error in $1 / N$, then it goes by a (short) photon noise regime in $1 / \sqrt{N}$ and eventually reaches for the brightest sources a plateau due to speckle noise - analog to the one known in the case of the visibility (Goodman 1985, Tatulli et al. 2004 ) - which limits the ultimate precision on the phase. Note already that in order to keep the phase noise below reasonable levels, that is smaller than 1 radian, it is mandatory to equip interferometers with $\mathrm{AO}$ systems, to at least insure a low-order correction of the turbulent phase providing Strehl ratios greater than $\sim 0.1$ for $D / r_{0}=8$ and $\sim 0.5$ for $D / r_{0}=2$.

Going in further details, Fig. 2 (bottom) shows the ratio of the error of the multimode phase by the error of the singlemode phase as a function of the number of photoevents. Clearly, two different behaviors occur whether we are considering photon-starved or photon rich regime, as discussed in the following.

\subsection{Low light level regime - limiting magnitude}

In the case of faint sources, the phase error is dominated by the detector noise $\sigma_{d e t_{\phi}}$. The comparison between both multimode and single-mode cases is however not straightforward because the number of pixels required to sample the fringes is specific to each technique, and depends on the chosen combination scheme. Let us first review here the possible technical solutions:

Multimode - (image plane) multiaxial combination: In this case, each speckle in which the interferograms are formed must be sampled correctly, that is it must be crossed by at least two pixels (Chelli \& Mariotti 1986). Then, if we take the whole image of size $\lambda / r_{0}$, the total number of pixels required becomes $N_{p i x}=2 D / r_{0}$. As a consequence, in such a combination mode, $N_{p i x}$ is dependent of the turbulence. In the case of the VLTI at Cerro Paranal, the average $r_{0}$ is $\simeq 1 \mathrm{~m}$ in the K-band, which gives turbulence strengths of $D / r_{0} \simeq 8$ and $D / r_{0} \simeq 1.8$ for the UTs $(D=8 \mathrm{~m})$ and ATs $(D=1.8 \mathrm{~m})$. This corresponds to a number of pixels that can vary quite a lot with the strength of the turbulence, respectively $N_{p i x}=16$ (UTs) and $N_{p i x}=4(\mathrm{ATs})$.

Multimode - (pupil plane) coaxial combination: Providing that the interferogram is scanned faster than the coherent time of the atmosphere in order to "freeze" the fringes, the minimum number of pixels to obtain full information on the temporal interferogram is 3 , which corresponds to the 3 degrees of freedom: incoherent flux, fringe amplitude, and phase. However, instead of this "ABC" scheme with 120 degrees between the three channels, it is frequently more practical to implement an "ABCD" scheme with 90 degree phase shifts, hence requiring 4 pixels. Note that in the framework of phase tracking, it is also possible to only measure the sine component of the fringe with "AC" scheme with 180 degrees between the two channels. In any case, the number of pixels needed in a coaxial pupil plane combiner is between 2 and 4 .

Single-mode - multiaxial combination: On the contrary of the multimode/multiaxial combination, the number of pixels is here independent of the turbulence as the shape and frequency of the interferogram are fixed by the design of the beam combiner. Typically, the interferogram consists in a sinusoidal signal with a frequency defined by the separation of the output pupils (so-called the coding frequency), and where its amplitude is modulated by the Gaussian envelope of the single-mode device. Tatulli \& LeBouquin (2006) have shown that the optimum number of pixels which respects the Shannon criterion $(>2$ pixels per fringe) and prevents from an overlapping of the photometric and interferometric peaks in the Fourier plane is $N_{p i x}=10$.

Single-mode - coaxial combination: As in the coaxial multimode case, one just need here to sample the interferogram with respect to the 3 degrees of liberty. As a 

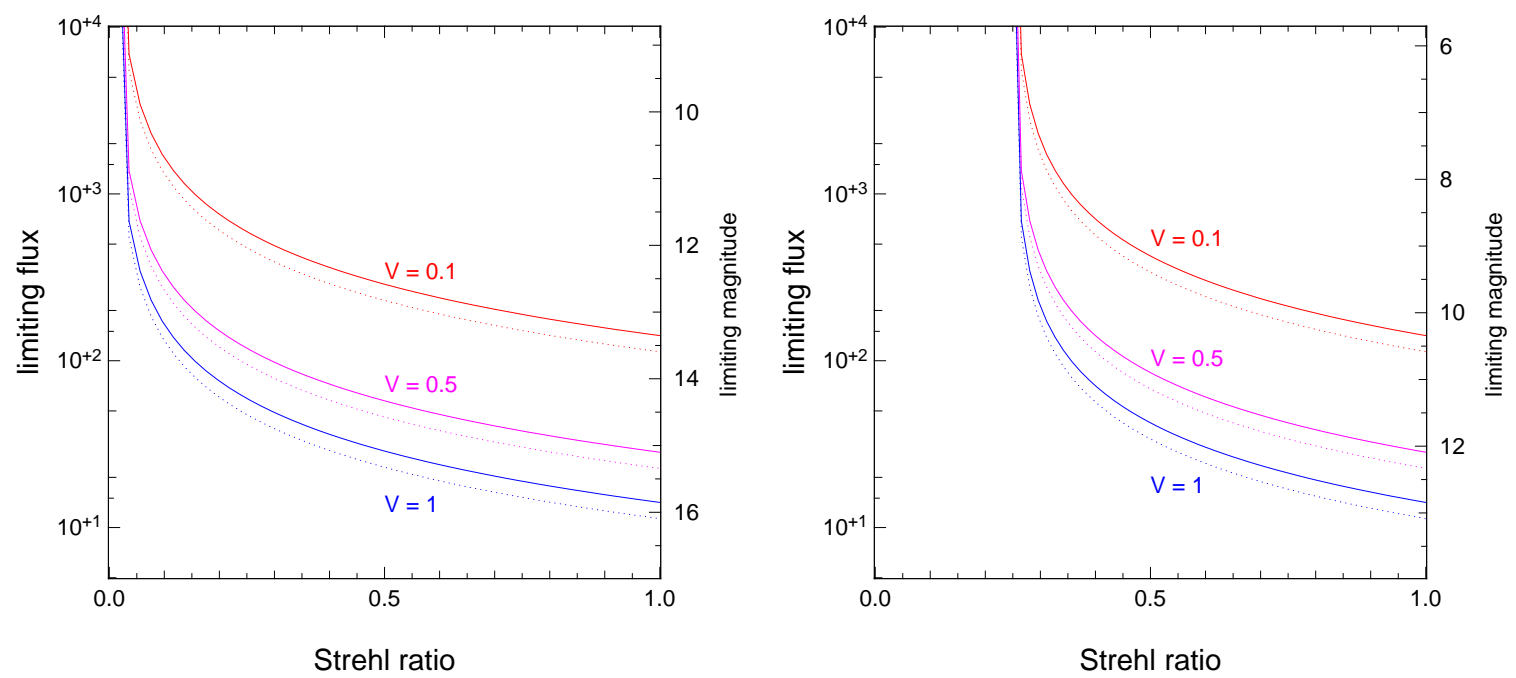

Fig. 3. Limiting flux as a function of the Strehl ratio (detector noise regime case), for turbulence strengths of $D / r_{0}=8$ (left) and $D / r_{0}=2$ (right). Plots are shown for an unresolved $(\mathrm{V}=1)$, fairly resolved $(\mathrm{V}=0.5)$ and fully resolved $(\mathrm{V}=0.1)$ source, respectively. Curves are going by pair, for single-mode (solid lines) and multimode (dotted lines) cases. The fixed parameters have the same values than previous figures.

result, the number of pixels is again between 2 and 4 . Note that instead of the usual temporal coding, fringes can be scanned simultaneously thanks to "ABCD-like" integrated chip devices (Benisty et al. 2009).

As a consequence, coaxial schemes - both in multimode and single-mode cases - appear more appropriate since they are using substantially less pixels than multiaxial ones. We remark however that these conclusions apply in the framework of fringe tracking where pair-wise combinations are favored. They may differ in the context of interferometric imaging instrument where an important number of baselines is involved. In the following we will consider coaxial combiners with $N_{p i x}=4$, corresponding to the standard "ABCD" sampling.

As multimode and single-mode fringe trackers finally require the same number of pixels, a straightforward comparison of the expressions of the detector noise shows that multimode combiners will achieve in this regime slightly better performance by a factor of $1 / \rho_{0}$, because single-mode spatial filters can not transmit $100 \%$ of the flux for the geometrical reasons mentioned in previous sections. If we define the limiting magnitude $\AA^{3}$ such as the error of the phase is equal to $1 \mathrm{rad}$, which - apart from very low AO correction levels - occurs in this detector noise regime, the corresponding limiting flux is given by:

$$
K_{\text {lim }}^{[\text {multi,single }]}=\frac{\sqrt{2} \sqrt{N_{\text {pix }}} \sigma_{d e t}}{\left[1, \rho_{0}\right] V_{12} \mathrm{e}^{-\sigma_{\phi_{r}}^{2}}}
$$

\footnotetext{
${ }^{3}$ note that the definition of the limiting magnitude depends on the estimator chosen to measure the phase. In this paper, the interferometric phase is merely estimated at the baseline frequency $f_{12}$, that is at the top of the interferometric peak (see Appendices A.3 and A.4. If a different estimator is used, like integrating the high frequency peak over the frequency range $\left[f_{12}-D / \lambda, f_{12}+D / \lambda\right]$ (see e.g. Roddier \& Lena 1984, Mourard et al. 1994, in the case of the squared visibility estimators) the expression, and therefore the value of the limiting magnitude will change accordingly.
}

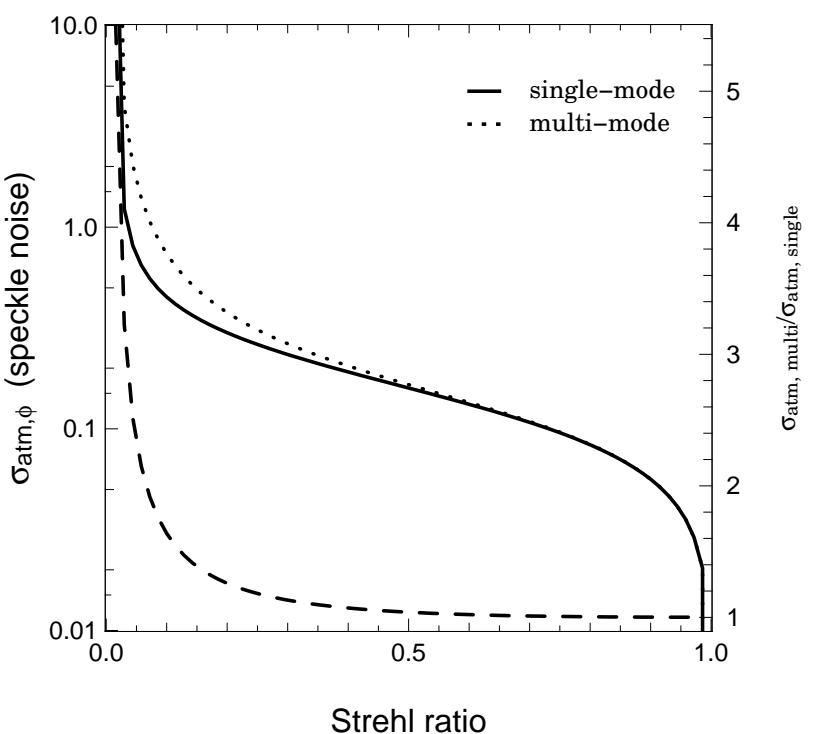

Fig. 4. Phase speckle noise as a function of the Strehl ratio for both single-mode (solid line) and multimode (dotted line) cases, with $D / r_{0}=8$. The ratio between the multimode and the single-mode phase errors is plotted in dashed line (y-axis on the right side).

with the factor 1 or $\rho_{0}$ depending whether we are using multimode or single-mode interferometers. As illustrated by Fig. 3, the gain in limiting magnitude for multimode combiners is $\sim 0.25$ magnitude.

\subsection{High light level regime: the speckle noise}

In the photon-rich regime, the speckle noise dominates and the error on the interferometric phase reaches a plateau, that is, is not dependent on the flux of the source anymore. As illustrated on Fig. 4, single-mode interferometers always provides in such a case lower phase error than multimode 
ones, emphasizing the remarkable properties of spatial filtering of the turbulent wavefront by single-mode devices. Such behavior has been already noticed by Tatulli et al. (2004) for the estimation of the squared visibility, showing that the so-called modal speckle noise of the visibility was, for a given AO correction, always smaller than classical speckle noise of multimode interferometers. The concept of modal speckle noise can also be applied for the singlemode interferometric phase. However at the difference of the squared visibility for which the modal speckle noise is 0 for a point source, the phase modal speckle noise always exists, independently of the size of the source.

Note that the gain of using single-mode schemes is all the more important than the the level of correction is low. If for fairly good correction with Strehl ratio above 20\%, the difference remains marginal with a factor $\sim 1-1.5$ between the single-mode and multimode phase error, the situations where bright sources are observed with low/none AO correction will highly benefit of single-mode interferometers. In such cases the precision of the phase can increase by at least a factor of 2 and much more when using spatial filtering of the corrugated wavefront with a typical Strehl ratio below $10 \%$. This is a typical counter-intuitive example where it is more profitable to loose a substantial part of the flux and keep only the coherent part of the perturbed wavefronts than conserving the whole flux at the price of introducing additional atmospheric noise.

\section{Application to fringe tracking}

In this section, we apply the formalism developed previously in the context of on-axis and off-axis fringe tracking, that is when the phase is estimated and compensated in real time to stabilize the fringes on the detector. We focus on the relative performance of fringe tracking systems using whether multimode or single-mode schemes.

\subsection{Coherent integration}

For each interferogram, fringes are shifted from the zero optical path difference by the turbulent piston. This piston is corrected in real time by the fringe tracker which estimates the phase of the fringes and compensates the optical path difference in real time by moving dedicated mirrors.

Centering the fringes in real time allows to perform coherent integration of the signal, that is to integrate on time scales much longer than the coherence time of the atmosphere. However, the estimated piston used for the opd correction is affected by a random measurement error $\epsilon(t)$. As a result, the interferogram is not perfectly centered and is still moving with an excursion depending on the statistics of the noise. If the interferograms are integrated over a time long enough for the realizations of the random error $\epsilon(t)$ to span all the range of its probability law, then, by rules of ergodicity, the visibility will be affected by a loss of contrast 4 which writes:

$$
<V>_{t}=<V>_{\epsilon}=V e^{-\frac{\sigma_{\phi}^{2}}{2}}
$$

\footnotetext{
${ }^{4}$ For sake of simplicity, we assume here that the phase is compensated instantaneously, hence not taking into account the delay of the fringe tracking loop between the measurement of the phase and its correction. The problem of time delay, which is independent of the multimode or single-mode nature of the fringetracking system, is treated in Conan et al. (2000).
}

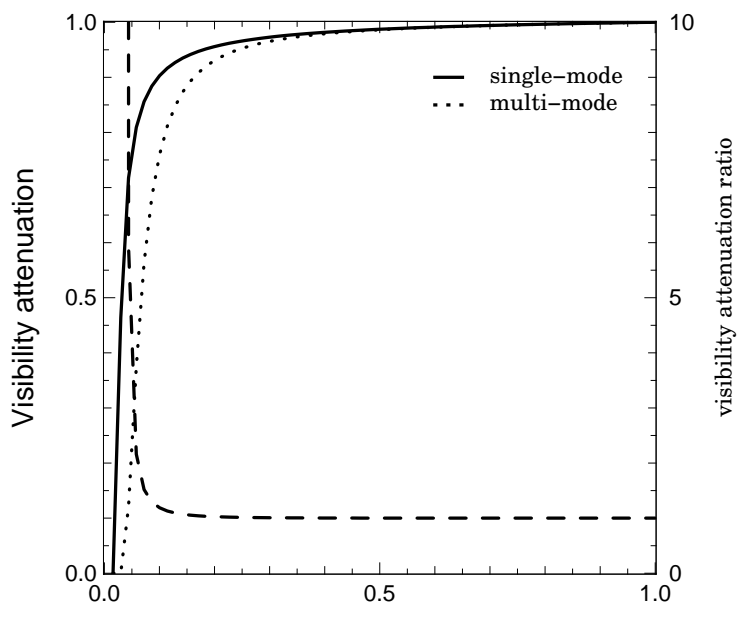

Strehl ratio

Fig. 5. Long exposure visibility attenuation due to the atmospheric noise of the measurement of the phase, as a function of the Strehl ratio for multimode (dotted line) and single-mode (solid line) systems, with $D / r_{0}=8$. The ratio of the attenuation between single-mode and multimode case is plotted in dashed line (y-axis on the right side).

where \langle\rangle$_{\epsilon}$ is the ensemble average over the realizations of the noise, and $\sigma_{\phi}^{2}$ is the variance of this noise as computed in Sect. 3.1. Hence, for long integration times, the fact that the instantaneous turbulent phase is corrected only to the precision of its estimation, introduces an attenuation of the coherent flux (equivalently, of the visibility) by a factor $e^{-\frac{\sigma_{\phi}^{2}}{2}}$. Such bias therefore depends on whether single-mode or multimode fringe tracking is used.

For high-light level regime in which fringe tracking is mostly expected to work, Fig. 5 shows the attenuation of the visibility as a function of the Strehl ratio for both single-mode and multimode systems. We can see that acceptable loss of contrast, typically $\gtrsim 0.8$, is achieved as soon as moderate $\mathrm{AO}$ corrections with Strehl $\gtrsim 0.1$ (for $D / r_{0}=8$ ) is provided. On the contrary, for lower performance of the AO system, the attenuation coefficient drops rapidly and the advantage of tracking the fringes is lost due to the uncompensated turbulent fluctuations of the phase over the pupils.

One important conclusion to draw is that, for a given $\mathrm{AO}$ correction, the visibility attenuation induced by the noise of the phase is always smaller when using single-mode fringe tracking systems instead of multimode ones. In other words, the maximum achievable atmospheric contrast is always higher using single-mode fringe tracker than multimode systems. This is especially true for low AO correction cases where the attenuation can becomes twice larger and more, as shown in Fig. 5

\subsection{Phase jumps}

It is usually implicitly assumed that the phase estimated in interferometry is effectively the shift of the fringes with respect to the zero opd, namely the piston phase. By definition, the piston phase shift is the average differential phase across the apertures, as illustrated on Fig. 1 and described 
E. Tatulli et al.: Multimode vs. single-mode interferometric phase
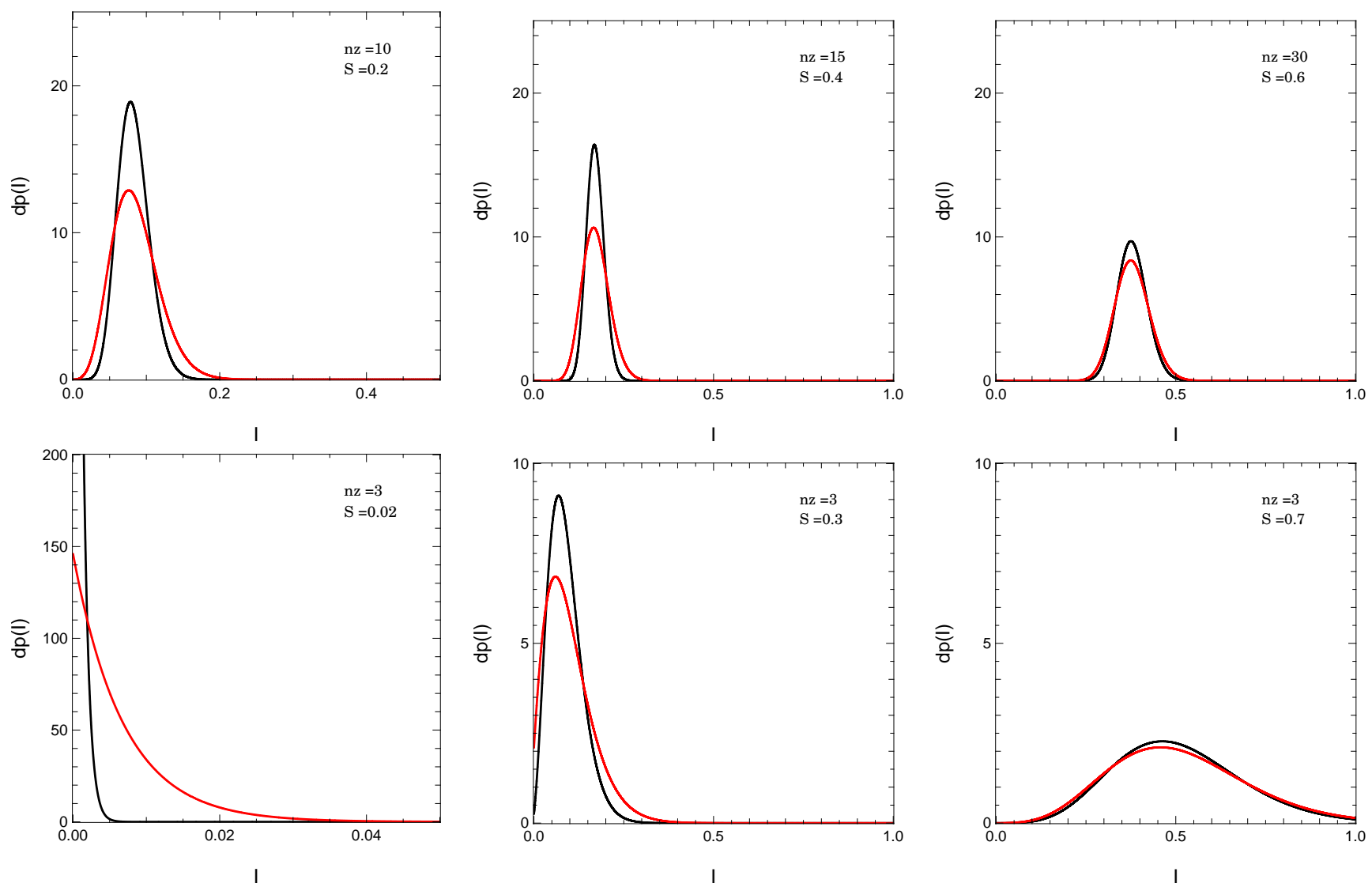

Fig. 6. Density probability of the multimode instantaneous Strehl ratio $\left(I=\left|T_{12}\right|^{2}\right.$, red lines $)$ and single-mode one $\left(I=\left|\rho_{12}\right|^{2}\right.$, black lines) for various levels of AO correction and turbulence strengths. Top: constant turbulence strength $D / r 0=8$ and the number of corrected Zernike are, from left to right, $n_{z}=10,15,30$. Bottom: constant AO correction $n_{z}=3$ (tip-tilt only), with various turbulence strengths, from left to right $D / r_{0}=8,4,2$.

in Eq. (1) that can be rewritten:

$$
\phi_{12}^{p}(t)=\int_{\Sigma}\left[\phi_{1}^{r}(\boldsymbol{r}, t)-\phi_{2}^{r}(\boldsymbol{r}, t)\right] \mathrm{d} \Sigma
$$

following the formalism of Buscher et al. (2008, see Eq. (6)), where $\mathrm{d} \Sigma$ is an elemental within the aperture $\Sigma$.

As first brought up by this author, the practical estimation $\widetilde{\phi_{12}^{p}}(t)$ of such piston phase is performed by taking the argument of the complex phasor averaged over the pupils, that is:

$$
\widetilde{\phi_{12}^{p}}(t)=\arg \left(\int_{\Sigma} e^{i\left(\phi_{1}^{r}(\boldsymbol{r}, t)-\phi_{2}^{r}(\boldsymbol{r}, t)\right)} \mathrm{d} \Sigma\right)
$$

in the multimode case, and:

$$
\widetilde{\phi_{12}^{p}}(t)=\arg \left(\int_{\Sigma} e^{i \phi_{1}^{r}(\boldsymbol{r}, t)} \mathrm{d} \Sigma \int_{\Sigma} e^{-i \phi_{2}^{r}(\boldsymbol{r}, t)} \mathrm{d} \Sigma\right)
$$

in the single-mode one.

By comparing Eq. (12) to Eqs. 13, 14) we can see that the true piston phase $\phi_{12}^{p}$ and the estimated one $\widetilde{\phi_{12}^{p}}$ are literally not the same, unless when there is no phase fluctuation (i.e. no turbulence or a perfect correction of the wavefronts) across the apertures.

When considering a small amount of fluctuations $\delta \phi_{12}(\boldsymbol{r}, t)$ such as $\phi_{1}^{r}(\boldsymbol{r}, t)-\phi_{2}^{r}(\boldsymbol{r}, t)=\delta \phi_{12}(\boldsymbol{r}, t)$, a Taylor expansion of Eqs. 13 14 can show that $\phi_{12}^{p}$ and $\widetilde{\phi_{1}^{p}}$ will then deviate roughly as $\int_{\Sigma}\left[\delta \phi_{12}(\boldsymbol{r}, t)\right]^{3} \mathrm{~d} \Sigma$ (Buscher et al. 2008). But if these fluctuations are large (typically above 1 radian), there can be strong differences between the piston phase and the argument of the complex phasor. More precisely, strong divergences are occurring when the complex phasor has a very small amplitude and eventually crosses the origin of the complex plane. In such cases, rapid phase jumps of the argument of the phasor can be experienced whereas these jumps are not seen in the piston phase. As a consequence the correction performed by the fringe tracker may be highly wrong and fringes potentially lost, especially if the science camera is working at a different wavelength than that of the fringe tracker (Buscher et al. 2008), as it is the case e.g. for the FINITO instrument (fringe tracking in H-band and correction in K-band, Gai et al. (2004); Le Bouquin et al. (2008)).

It is therefore important to know the probability of phase jumps to occur in order to ensure an observational/instrumental context in which these events are avoided as much as possible. So far, the conclusions about this point are not clear : if Tubbs (2005) implied that spatial filtering could be the source of such anomalies, Buscher et al. (2008) on the contrary argues that AO correction and most of all spatial filtering help to reduce these singularities. All the previous analysis were however based only on simulations. We propose here a theoretical analysis of this 
phenomenon. We emphasize that our analysis focuses on the rate of occurrence of phase jumps, as defined above. We do not study the consequences of these phase jumps in terms of effective loss of fringe tracking. Such a causality will depend on the system (e.g. single wavelength vs. multiple spectral channels methods) used to practically estimate and correct the opd. Considering that a phase jump would systematically induce a failure in the fringe tracking system therefore corresponds to the worst case scenario.

The fact that the coherent flux drops to zero because of turbulent phase fluctuation depends on whether the interferometric transfer function $\left|T_{12}\right|$ for multimode systems or the interferometric coupling coefficient $\left|\rho_{12}\right|$ in the singlemode case, drops to zero. We thus want here to establish the probability density of these quantities, and study how likely they can take very small values. Canales \& Cagigal (1999) have studied the distribution of speckle statistics in presence of partial AO correction for mono-pupil telescopes. They have shown that the density probability $d p(I)$ of the intensity I at the center of the image - that is by definition, the instantaneous Strehl ratio - follows a Rician statistics of the form:

$$
d p(I)=\frac{1}{2 \sigma^{2}} \exp \left(-\frac{I+a^{2}}{2 \sigma^{2}}\right) I_{0}\left(-\frac{a \sqrt{I}}{\sigma^{2}}\right)
$$

where $a^{2}$ and $\sigma^{2}$ depends on the first and second order moments of the real and imaginary part of the complex phasor describing the AO corrected turbulent wavefront. Note that such analytical definition of the density probability implicitly assumes a time-constant $r_{0}$ to characterize the turbulence. This hypothesis is the limitation of our model as $r_{0}$ may actually vary on time scales of minutes and shorter, driving to brief episodes of very small $r_{0}$ that would trip up AO systems and lead to very small Strehl ratio for a brief period of time. However, modelling this effect requires more complex and heavy simulations of partially AO-corrected turbulence which are out out the scope of this paper.

We have emphasized in Sect. 2 that $\left|T_{12}\right|^{2}$ and $\left|\rho_{12}\right|^{2}$ represent the instantaneous interferometric Strehl ratio, for the multimode and single-mode cases, respectively. Therefore, by straightforward analogy with an interferometric pupil instead of a monolithic one, we can show that $I=\left|T_{12}\right|^{2}$ and $I=\left|\rho_{12}\right|^{2}$ are also following a Rician distribution. The parameters $a^{2}$ and $\sigma^{2}$ relative to both instantaneous interferometric Strehl ratio can be directly derived from the formalism of previous section and their expressions are given in Table B.1 of Appendix B

Figure 6 shows the density probability of $\left|T_{12}\right|^{2}$ and $\left|\rho_{12}\right|^{2}$ for various levels of $\mathrm{AO}$ correction and turbulence strength.

High-order AO correction: First, we can see that, as soon as moderate AO correction is applied - roughly a few tens of modes or a Strehl ratio $\gtrsim 0.3$, the probability density displays a bell shape, all the more narrow than the AO correction is high, and the probability that $\left|T_{12}\right|$ or $\left|\rho_{12}\right|$ goes to very low values is null (typically, for $n_{z}=15$ and $D / r 0=8$, the probability for the interferometric instantaneous Strehl ratio to be $<0.01$ is $<10^{-9}$, and $<0.05$ is $<0.02 \%$ ), emphasizing again the relevance of associating high-performance $\mathrm{AO}$ systems to fringe tracking devices, the wavefront being spatially filtered or not. We note that the intensity mean value $\left.(<I\rangle=2 \sigma^{2}+a^{2}\right)$ is systematically slightly higher in the single-mode case and the dispersion $\left(\sigma_{I}^{2}=2 \sigma^{2}+4 \sigma^{2} a^{2}\right)$ slightly smaller. The difference is however not critical.

Low-order AO correction: For low-order AO such as tip-tilt correction only, the situation is fairly different. The shape of the distribution has changed, peaking at zero in the case of strong turbulence with high values of $D / r_{0} \gtrsim 5$. The probability to have a very small value of the intensity is thus significant, and events such as phase jumps are likely to happen. In other words, tip-tilt correction is a too low order correction to prevent from such events.

Figure 7 displays the probability $P(I)$ for the instantaneous interferometric Strehl ratio to reach values close to zero (i.e. $P(I<\epsilon)$ with $\epsilon$ very small) for different strengths of the atmospheric turbulence. We can see that, for strong turbulence $\left(D / r_{0} \gtrsim 5\right)$, the probability $P(I)<\epsilon$ is always higher in the single-mode case. In such cases, we expect phase jumps to occur more frequently with single-mode fringe tracker, as the likelihood that two speckles are simultaneously entering the single-mode fibers of the two telescopes is weak. However, this does not mean that phase jumps should not occur often with multimode fringe tracker too. As an example the probability to have instantaneous interferometric Strehl lower than 0.01 in the multimode case is $\sim 78 \%$ for $D / r_{0}=8$, whereas it is $100 \%$ in the single-mode one. In other words, regardless of the multimode versus single-mode issue, one should never consider to perform fringe tracking with big telescope apertures associated with the sole tip-tilt correction.

For weak turbulence (roughly $D / r_{0} \lesssim 5$, Fig. 6. bottom middle and right), the distribution starts to look Tike a bell shape again, with however non null probability to have a zero intensity. But as shown in Fig. 7 this probability is now higher in the multimode case, as only a few and big speckles are present in the images. As a matter of fact, this case is the one treated by Buscher et al. (2008, see Fig. 4, with $D / r_{0}=4$, tip-tilt correction) in his simulations, and we come to the same conclusion than his: in these conditions, spatial filtering enables to decrease the number of phase jump events. We however emphasize here that this statement is true only for cases of moderate turbulence strength.

Application to the VLTI: We recall that at Paranal the average $r_{0}$ is $\sim 1 \mathrm{~m}$ in the $\mathrm{K}$-band, which gives turbulence strength of $D / r_{0} \sim 8$ and $D / r_{0} \sim 1.8$ for the UTs and ATs, respectively. UTs come with high-order Adaptive Optics systems (MACAO, Arsenault et al. 2004) therefore one can equally choose multimode or single-mode fringe tracking schemes since (i) phase jumps are unlikely to occur in any case, and (ii) multimode and single-mode fringe trackers will provide the same robustness to eventual phase jumps. In the case of ATs where tip-tilt correction is currently provided, the likelihood to endure phase jumps is also very low. However to maximize the stability of their fringe tracking system, it still seems appropriate to accompany the ATs with single-mode spatial filtering devices as the probability to undergo such phase jumps remains roughly 10 times lower (see Fig. 7, right). Alternatively, providing higher order AO correction to ATs will fix the phase jump issue. 

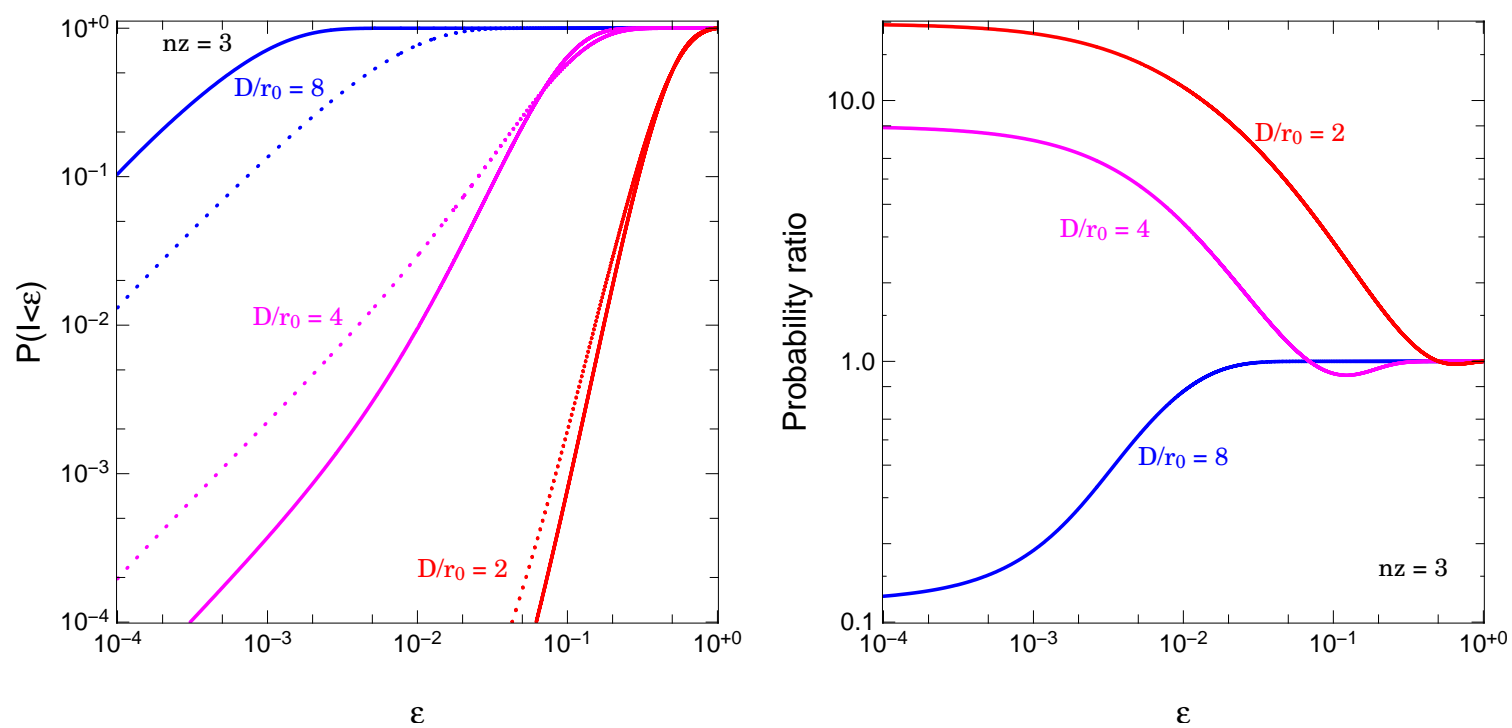

Fig. 7. Left: probability for the instantaneous interferometric Strehl ratio I to be lower than the value $\epsilon$, in the case of tip-tilt correction only, for different strengths of turbulence $D / r_{0}=8,4,2$ (respectively in blue, magenta and red), for the multimode (dotted line) and single-mode (solid line) cases. Right: Multimode versus single-mode probability ratio.
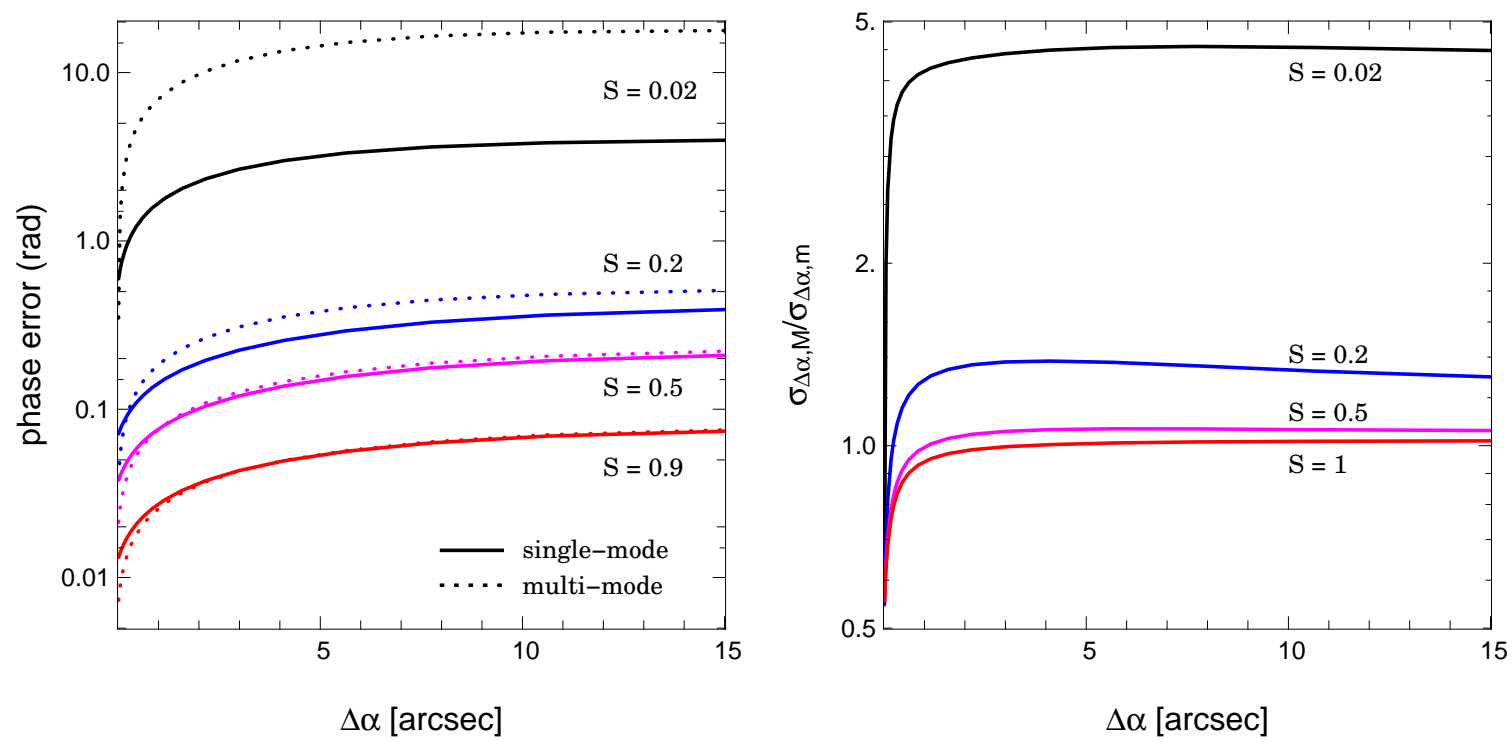

Fig. 8. Left: astrometric phase error due to atmospheric turbulence, as the function of the angular distance $\boldsymbol{\Delta} \alpha$ between the source and the reference star, for both single-mode (solid lines) and multimode (dotted lines). Right: ratio of the multimode versus the single-mode astrometric error. Results are shown for various AO correction levels as described in previous Figures. We have assumed one single turbulent layer located at $h=10 \mathrm{~km}$.

\subsection{Phase referencing - astrometry}

The interest of phase referencing instruments such as PRIMA recently installed on the VLTI (Delplancke et al. 2000 ), is (i) to provide sub-microarcsecond precision astrometry, allowing e.g. to detect the presence of a faint companion (extrasolar planet) around the central star (Launhardt et al. 2008), and (ii) to drastically increase the limiting magnitude of the interferometer by locking the fringes of the (possibly faint) science object on a simultaneously observed bright off-axis reference star whose phase is used as reference (Sahlmann et al. 2008). Such method requires that the nearby reference star is close enough - typically in the isoplanatic patch - in order to assume that both the wavefront of the source and the reference are identically perturbed by the atmospheric turbulence. Strictly speaking, this assumption is not true as the optical paths of the two stars are different, crossing different part of the atmosphere in the turbulent layers. As a result, the loss of correlation between the two wavefronts will drive to an atmospheric noise on the astrometric phase which will lower the ultimate performance of this method.

We call respectively $\phi_{s}$, and $\phi_{r}^{\Delta \alpha h}$, the phase of the astrophysical target and the phase estimated from the off-axis reference source, located at an angular distance $\Delta \alpha$ of the science object, and where we assume for the sake of sim- 
plicity a single turbulent layer located at an height $h$ from the ground. The astrometric phase is then simply defined by:

$$
\Delta_{\phi}^{\Delta \alpha h}=\phi_{s}-\phi_{r}^{\Delta \alpha h}
$$

and its associated error writes:

$$
\sigma_{\Delta_{\phi}^{\Delta \alpha h}}^{2}=2 \sigma_{\phi}^{2}-2 \operatorname{cov}\left(\phi_{s}, \phi_{r}^{\Delta \alpha h}\right)
$$

Equation 17 tells us that the noise of the astrometric phase increases as long as the correlation between the two turbulent wavefronts $\operatorname{cov}\left(\phi_{s}, \phi_{r}^{\Delta \alpha h}\right)$ decreases, that is as long as the spatial distance $\Delta \alpha h$ between both turbulent phase screens is getting higher. The quantitative effect of the separation between the star and its reference on the interferometric narrow-angle astrometric error has been already studied by Shao \& Colavita (1992) and we refer the readers to their paper for further details. In our analysis, we focus on the relative performance of astrometry between multimode and single-mode scheme, studying how spatial filtering of the wavefronts will impact on the astrometric error, as developed in Appendix C. For sake of simplicity we have assumed in the following one single turbulent layer located at $h=10 \mathrm{~km}$ as it is the strongest layer at the Cerro Paranal site (Masciadri et al. 1999). There is also a strong layer very close to the ground at around 20m (Martin et al. 2000) but that only marginally contributes to the phase decorrelation since the linear distance at stake is 500 times smaller than the one relative to the high altitude layer. Figure 8 (left) shows the evolution of the astrometric error as a function of the angular distance between the source and the reference, for both single-mode and multimode schemes.

Large field of view: When the reference source is further than roughly $\simeq 1^{\prime \prime}$ from the astrophysical object of interest, we can see that spatial filtering allows to increase the precision of the astrometric phase. The improvement is better as the $\mathrm{AO}$ correction is low, reaching a factor of $\sim 4$ without AO correction, as emphasized by Fig. 8 (right). This argues in favor of using spatial-filtering elements in the design of astrometric instruments with large field of view (FOV) as it has been chosen for PRIMA $\left(\mathrm{FOV} \simeq 30^{\prime \prime}\right)$. Looking in further details, one can notice that the phase tends asymptotically towards a plateau, from an angular separation of $\sim 10$ " which roughly corresponds to the isoplanatic angle of Paranal in the K-band ${ }^{5}$ Indeed from this angle, wavefronts can be considered as uncorrelated and the astrometric error converges to $\sqrt{2} \sigma_{\phi}$, which does not depend on the separation $\Delta \alpha$ any longer. As a consequence in this regime (i.e. $\Delta \alpha \gtrsim 10^{\prime \prime}$ ), the astrometric phase error ratio can be approximated by the speckle phase error ratio, which has already been discussed in Sect. 3.3

Narrow field of view: At the contrary, when wavefronts are still strongly correlated $\left(\Delta \alpha \lesssim 1^{\prime \prime}\right)$, the performance of single-mode astrometry is slightly lower (by a factor $\sim 1$ to $\sim 1.5$ ) than that of multimode astrometry, the

\footnotetext{
${ }^{5}$ considering the measurements of Martin et al. (2000) which have found an average isoplanatic angle of $\sim 1.9^{\prime \prime}$ in the visible, and recalling that it evolves with wavelength as $\lambda^{1.2}$ (Shao \& Colavita 1992).
}

error of the latter decreasing faster as the separation between the astrophysical source and the reference shrinks to zero. By smoothing the turbulent wavefronts across the apertures, spatial filtering is indeed lowering the effect of the strong correlation between the wavefronts. Such situation concerns the cases for which the reference is very close to the star, like GRAVITY the second generation of astrometric instruments of the VLTI (Gillessen et al. 2006, $\mathrm{FOV} \simeq 2^{\prime \prime}$ )

\section{Summary and conclusions}

In this paper, we have provided a theoretical formalism that allows to derive the error of the interferometric phase, both in cases of single-mode and multimode interferometry. From these derivations, we have demonstrated that:

- Contrarily to a widespread idea, losing flux by injecting the light into single-mode spatial filters is not a performance killer for estimating the phase. As the matter of fact, single-mode interferometry provides better performance than that of multimode interferometry, unless when the interferometer is working in the detector noise regime (faint sources). In such cases, multimode interferometry is slightly better, providing a phase error smaller by a factor $\rho_{0} \simeq 0.8$ which is the maximum fraction of flux that can be injected in single-mode devices.

- In cases of bright sources observations, spatial filtering is shown to be very efficient, especially when the AO correction of the turbulent wavefronts is poor or absent. In such situations, the precision of the single-mode interferometric phase is better than that of the multimode one by a factor of 2 and more when the Strehl is below $10 \%$.

- Single-mode interferometry also proves to be more robust to the turbulence to both locking and coherently integrating the fringes and providing a better astrometric precision when using phase referencing techniques, except for narrow field of views $\left(\mathrm{FOV} \lesssim 1^{\prime \prime}\right)$.

In conclusion, from a theoretical point of view and contrarily to a widespread opinion, single-mode fringe tracking should be seriously considered as an advantageous technical solution. Furthermore, the astronomers should realize that the many gains of single-mode interferometry (flexibility of the solutions, robustness to the alignment, less optical elements,...) may compensate significantly a modest and limited loss of performance in the faint sources case compared to multimode interferometry solutions. This is all the more true that the detectors should soon evolve to reach photon counting capability.

\section{References}

Arsenault, R., Donaldson, R., Dupuy, C., et al. 2004, in Society of Photo-Optical Instrumentation Engineers (SPIE) Conference Series, Vol. 5490, Society of Photo-Optical Instrumentation Engineers (SPIE) Conference Series, ed. D. Bonaccini Calia, B. L. Ellerbroek, \& R. Ragazzoni, 47-58

Beckers, J. M. \& Hege, E. K. 1984, in IAU Colloq. 79: Very Large Telescopes, their Instrumentation and Programs, ed. M.-H. Ulrich \& K. Kjaer, 279-293

Benisty, M., Berger, J., Jocou, L., et al. 2009, A\&A, 498, 601

Berger, D. H., Monnier, J. D., Millan-Gabet, R., et al. 2008, in Presented at the Society of Photo-Optical Instrumentation Engineers (SPIE) Conference, Vol. 7013, Society of Photo-Optical Instrumentation Engineers (SPIE) Conference Series 
Buscher, D. 1988, MNRAS, 235, 1203

Buscher, D. F., Young, J. S., Baron, F., \& Haniff, C. A. 2008, in Presented at the Society of Photo-Optical Instrumentation Engineers (SPIE) Conference, Vol. 7013, Society of Photo-Optical Instrumentation Engineers (SPIE) Conference Series

Canales, V. F. \& Cagigal, M. P. 1999, Appl. Opt., 38, 766

Chelli, A. 1989, A\&A, 225, 277

Chelli, A. \& Mariotti, J. M. 1986, A\&A, 157, 372

Colavita, M. M. 1999, PASP, 111, 111

Conan, J. 1994, PhD thesis, Ph. D. thesis, Université Paris XI Orsay, (1994)

Conan, R., Ziad, A., Borgnino, J., Martin, F., \& Tokovinin, A. A. 2000, in Presented at the Society of Photo-Optical Instrumentation Engineers (SPIE) Conference, Vol. 4006, Society of Photo-Optical Instrumentation Engineers (SPIE) Conference Series, ed. P. Léna \& A. Quirrenbach, 963-973

Coudé du Foresto, V., Faucherre, M., Hubin, N., \& Gitton, P. 2000, A\&AS, 145,305

Coudé du Foresto, V., Ridgway, S., \& Mariotti, J.-M. 1997, A\&AS, 121,379

Delplancke, F., Leveque, S. A., Kervella, P., Glindemann, A., \& D'Arcio, L. 2000, in Presented at the Society of Photo-Optical Instrumentation Engineers (SPIE) Conference, Vol. 4006, Society of Photo-Optical Instrumentation Engineers (SPIE) Conference Series, ed. P. Léna \& A. Quirrenbach, 365-376

Dyck, H. M., Benson, J. A., Carleton, N. P., et al. 1995, AJ, 109, 378

Fried, D. L. 1965, Journal of the Optical Society of America (19171983), 55, 1427

Fusco, T. \& Conan, J.-M. 2004, Journal of the Optical Society of America A, 21, 1277

Gai, M., Menardi, S., Cesare, S., et al. 2004, in Presented at the Society of Photo-Optical Instrumentation Engineers (SPIE) Conference, Vol. 5491, Society of Photo-Optical Instrumentation Engineers (SPIE) Conference Series, ed. W. A. Traub, 528-+

Gillessen, S., Perrin, G., Brandner, W., et al. 2006, in Presented at the Society of Photo-Optical Instrumentation Engineers (SPIE) Conference, Vol. 6268, Society of Photo-Optical Instrumentation Engineers (SPIE) Conference Series

Goodman, J. W. 1985, Statistical optics (New York, WileyInterscience, 1985,567 p.)

Jurgenson, C. A., Santoro, F. G., Baron, F., et al. 2008, in Presented at the Society of Photo-Optical Instrumentation Engineers (SPIE) Conference, Vol. 7013, Society of Photo-Optical Instrumentation Engineers (SPIE) Conference Series

Launhardt, R., Queloz, D., Henning, T., et al. 2008, in Presented at the Society of Photo-Optical Instrumentation Engineers (SPIE) Conference, Vol. 7013, Society of Photo-Optical Instrumentation Engineers (SPIE) Conference Series

Le Bouquin, J.-B., Abuter, R., Bauvir, B., et al. 2008, in Presented at the Society of Photo-Optical Instrumentation Engineers (SPIE) Conference, Vol. 7013, Society of Photo-Optical Instrumentation Engineers (SPIE) Conference Series

Martin, F., Conan, R., Tokovinin, A., et al. 2000, A\&AS, 144, 39

Masciadri, E., Vernin, J., \& Bougeault, P. 1999, A\&AS, 137, 203

Mège, J.-M. 2002, PhD thesis, Ph. D. thesis, (2002)

Mège, P., Malbet, F., \& Chelli, A. 2003, in Presented at the Society of Photo-Optical Instrumentation Engineers (SPIE) Conference, Vol. 4838, Society of Photo-Optical Instrumentation Engineers (SPIE) Conference Series, ed. W. A. Traub, 329-337

Mourard, D., Tallon-Bosc, I., Rigal, F., et al. 1994, A\&A, 288, 675

Noll, R. J. 1976, Journal of the Optical Society of America (19171983), 66, 207

Roddier, F. 1979, Journal d'Optique, 10, 299

Roddier, F. \& Lena, P. 1984, Journal of Optics, 15, 171

Sahlmann, J., Abuter, R., Di Lieto, N., et al. 2008, in Presented at the Society of Photo-Optical Instrumentation Engineers (SPIE) Conference, Vol. 7013, Society of Photo-Optical Instrumentation Engineers (SPIE) Conference Series

Shaklan, S. \& Roddier, F. 1988, Appl. Opt., 27, 2334

Shao, M. \& Colavita, M. M. 1992, A\&A, 262, 353

Tatulli, E. \& Chelli, A. 2005, Journal of the Optical Society of America A, 22, 1589

Tatulli, E. \& LeBouquin, J. 2006, MNRAS, 368, 1159

Tatulli, E., Mège, P., \& Chelli, A. 2004, A\&A, 418, 1179

Tatulli, E., Millour, F., Chelli, A., et al. 2007, A\&A, 464, 29

Tubbs, R. 2005, Appl. Opt., 44, 6253

Vannier, M., Petrov, R. G., Lopez, B., \& Millour, F. 2006, MNRAS, 367,825 


\section{Appendix A: Computation of the interferometric phase error}

\section{A.1. General formalism}

It follows Goodman's approach (Goodman 1985), based on a continuous model of detection process, where the recorded signal (i.e. the interferogram) can be represented as:

$$
d(x, y)=\sum_{k=1}^{K} \delta\left(x-x_{k}, y-y_{k}\right)
$$

and its Fourier transform:

$$
D(\boldsymbol{f})=D\left(f_{x}, f_{y}\right)=\sum_{k=1}^{K} \mathrm{e}^{-2 i \pi\left(f_{x} x_{k}+f_{y} y_{k}\right)}
$$

where the position $\left(x_{n}\right)$ and the number of photoevents (per time unit) $(K)$ are statistical processes with probability laws depending on the intensity distribution $I(\boldsymbol{x})$. To take also into account the detector noise, one needs to add Gaussian additive noise $(\epsilon)$ to the previous equation (Tatulli et al. 2004; Tatulli \& Chelli 2005):

$$
Q(\boldsymbol{f})=D(\boldsymbol{f})+\epsilon(\boldsymbol{f})=\sum_{k=1}^{K} \mathrm{e}^{-2 i \pi\left(f_{x} x_{k}+f_{y} y_{k}\right)}+\epsilon(\boldsymbol{f})
$$

Note that this formalism is also valid for a temporal coding of the interferogram, that is replacing the spatial position $\left(x_{k}, y_{k}\right)$ by the (1-D) temporal sampling $t$, and similarly the spatial frequency $\boldsymbol{f}$ by the temporal one $\nu$.

As $Q$ represents the spectrum of the interferogram, the phase of the spectrum is merely the argument of this estimator:

$$
\phi=\operatorname{atan}(\operatorname{Im}(Q), \operatorname{Re}(Q))
$$

In this framework, Chelli (1989) has shown that in first approximation (i.e. small noise error), the variance of the phase can be expressed as following:

$$
\sigma_{\phi}^{2}=\frac{1}{2} \frac{\mathrm{E}\left(|Q|^{2}\right)-\operatorname{Re}\left[\mathrm{E}\left(Q^{2}\right)\right]}{[\mathrm{E}(Q)]^{2}}
$$

then using Goodman's formalism described above, one can show that (Chelli 1989; Tatulli et al.|2004):

$$
\begin{aligned}
\mathrm{E}\left(|Q|^{2}\right) & =<\bar{K}^{2}|i(\boldsymbol{f})|^{2}+\bar{K}+N_{\text {pix }} \sigma_{\text {det }}^{2}>_{\Phi} \\
\mathrm{E}\left(Q^{2}\right) & =<\bar{K}^{2} i^{2}(\boldsymbol{f})>_{\Phi} \\
\mathrm{E}(Q) & =<\bar{K} i(\boldsymbol{f})>_{\Phi}
\end{aligned}
$$

where $\bar{K}$ is the average number of photoevents per time unit, $\sigma_{d e t}$ is the detector noise and $N_{\text {pix }}$ is the number of pixel to sample the signal. $i(\boldsymbol{f})$ is the normalized spectrum of the interferogram (i.e. $i(\mathbf{0})=1$ ).

$<>_{\Phi}$ denotes the expected value with respect to the atmosphere. How the previous expressions are unfolding depends on whether we deal with multimode or single-mode interferometry.

\section{A.2. Useful definitions}

In this section we introduce the concepts and notations that we will use in our formalism to derive the expression of the noise of the interferometric phase. Let us consider (see also Fig. 1 in the paper):

- an interferometer made of 2 telescopes described by their pupil functions $P_{1}(\boldsymbol{r}), P_{2}(\boldsymbol{r})$ and their associated transmission $\sqrt{t_{1}}, \sqrt{t_{2}}$. Note that with such a definition $P_{1}$ and $P_{2}$ are respectively centered at the position $\boldsymbol{r}_{1}=\lambda \boldsymbol{f}_{1}$ and $\boldsymbol{r}_{2}=\lambda \boldsymbol{f}_{2}$, and we introduce the quantity $\boldsymbol{f}_{12}=\boldsymbol{f}_{2}-\boldsymbol{f}_{1}$ as the baseline frequency of the interferometer.

- $\phi_{1}^{r}(\boldsymbol{r})$ and $\phi_{2}^{r}(\boldsymbol{r})$ the partially AO-corrected atmospheric turbulent phase screens. We also introduce $\mathcal{D}_{\phi_{r}}$ the structure function of the residual phases (assuming the same level of AO correction for each telescope) together with $\sigma_{\phi_{r}}^{2}$, its associated residual phase variance (see also Appendix D.

- the incoming wavefront of the source $\Psi(\boldsymbol{r})$. By definition we have $|\Psi(\mathbf{0})|^{2}=N, N$ being the number of photons per surface unit and per time unit emitted by the source, and $\left[\Psi(\boldsymbol{r}) \Psi^{*}(\boldsymbol{r}+\lambda \boldsymbol{f})\right] /|\Psi(\mathbf{0})|^{2}=V(\boldsymbol{f})$ the visibility of the source at the spatial frequency $\boldsymbol{f}$.

We emphasized that the following derivations will be done assuming monochromatic interferograms at the wavelength $\lambda$. Indeed, taking into account a non-null spectral bandwidth $\delta \lambda$ is equivalent to consider a monochromatic interferogram at the effective wavelength $\lambda_{0}$ of the filter, modulated in amplitude by an envelope, which width is fixed by the spectral coherence length $L_{c}=\lambda_{0}^{2} / \delta \lambda$. Moreover, such effect is independent of the multimode or single-mode nature of the interferogram. 


\section{A.3. Phase noise in multimode interferometry}

In the following, we derive the estimator of the coherent flux of the interferogram, and subsequently the error of the interferometric phase in the multimode case. We provide two formalisms that respectively describe both beam recombination schemes: in the image plane and in the pupil plane.

\section{A.3.1. Image plane recombination}

Image plane recombination is well suited to describe multiaxial interferometric schemes where the fringe pattern is spatially coded on the detector, such as for the GI2T and VEGA/CHARA instruments. Note that co-axial (i.e. temporal) coding can also be performed in the image plane but in practice - to our knowledge - no interferometric instruments are using this technique.

In such case, the spatial distribution of the complex amplitude of the radiation field $E(\boldsymbol{r})$ can be written as following:

$$
E(\boldsymbol{r})=\sqrt{t_{1}} P_{1}(\boldsymbol{r}) \exp ^{i \phi_{1}^{r}(\boldsymbol{r})} \Psi(\boldsymbol{r})+\sqrt{t_{2}} P_{2}(\boldsymbol{r}) \exp ^{i \phi_{2}^{r}(\boldsymbol{r})} \Psi(\boldsymbol{r})
$$

By rules of diffraction theory, the Fourier Transform of the interference pattern formed in the image plane is the autocorrelation of the complex amplitude $E(\boldsymbol{r})$, namely:

$$
\begin{aligned}
I(\boldsymbol{f}) & =\int E(\boldsymbol{r}) E^{*}(\boldsymbol{r}+\lambda \boldsymbol{f}) \mathrm{d} \boldsymbol{r} \\
& =t_{1} \int P_{1}(\boldsymbol{r}) P_{1}(\boldsymbol{r}+\lambda \boldsymbol{f}) \exp ^{i\left(\phi_{1}^{r}(\boldsymbol{r})-\phi_{1}^{r}(\boldsymbol{r}+\lambda \boldsymbol{f})\right)} \Psi(\boldsymbol{r}) \Psi^{*}(\boldsymbol{r}+\lambda \boldsymbol{f}) \mathrm{d} \boldsymbol{r} \\
& +t_{2} \int P_{2}(\boldsymbol{r}) P_{2}(\boldsymbol{r}+\lambda \boldsymbol{f}) \exp ^{i\left(\phi_{2}^{r}(\boldsymbol{r})-\phi_{2}^{r}(\boldsymbol{r}+\lambda \boldsymbol{f})\right)} \Psi(\boldsymbol{r}) \Psi^{*}(\boldsymbol{r}+\lambda \boldsymbol{f}) \mathrm{d} \boldsymbol{r} \\
& +\sqrt{t_{1} t_{2}} \int P_{1}(\boldsymbol{r}) P_{2}(\boldsymbol{r}+\lambda \boldsymbol{f}) \exp ^{i\left(\phi_{1}^{r}(\boldsymbol{r})-\phi_{2}^{r}(\boldsymbol{r}+\lambda \boldsymbol{f})\right)} \Psi(\boldsymbol{r}) \Psi^{*}(\boldsymbol{r}+\lambda \boldsymbol{f}) \mathrm{d} \boldsymbol{r} \\
& +\sqrt{t_{1} t_{2}} \int P_{1}(\boldsymbol{r}+\lambda \boldsymbol{f}) P_{2}(\boldsymbol{r}) \exp ^{i\left(\phi_{2}^{r}(\boldsymbol{r})-\phi_{1}^{r}(\boldsymbol{r}+\lambda \boldsymbol{f})\right)} \Psi^{*}(\boldsymbol{r}+\lambda \boldsymbol{f}) \Psi(\boldsymbol{r}) \mathrm{d} \boldsymbol{r}
\end{aligned}
$$

Introducing $|\Psi(\mathbf{0})|^{2}=N$ in previous equation enables to make the complex visibility function appear:

$$
I(\boldsymbol{f})=N t_{1} V(\boldsymbol{f}) S_{1}(\boldsymbol{f})+N t_{2} V(\boldsymbol{f}) S_{2}(\boldsymbol{f})+N \sqrt{t_{1} t_{2}} V(\boldsymbol{f}) S_{12}(\boldsymbol{f})+N \sqrt{t_{1} t_{2}} V(\boldsymbol{f}) S_{12}^{*}(-\boldsymbol{f})
$$

with $S_{1}(\boldsymbol{f})$ and $S_{2}(\boldsymbol{f})$ the so-called photometric peaks resulting on the autocorrelation of each corrugated pupil, and $S_{12}(\boldsymbol{f})$ the interferometric peak arising from the cross-correlation between both pupils:

$$
\begin{aligned}
S_{i}(f) & =\int P_{i}(\boldsymbol{r}) P_{i}(\boldsymbol{r}+\lambda \boldsymbol{f}) \exp ^{i\left(\phi_{i}^{r}(\boldsymbol{r})-\phi_{i}^{r}(\boldsymbol{r}+\lambda \boldsymbol{f})\right)} \mathrm{d} \boldsymbol{r}, \quad(\mathrm{i}=[1,2]) \\
S_{12}(f) & =\int P_{1}(\boldsymbol{r}) P_{2}(\boldsymbol{r}+\lambda \boldsymbol{f}) \exp ^{i\left(\phi_{1}^{r}(\boldsymbol{r})-\phi_{2}^{r}(\boldsymbol{r}+\lambda \boldsymbol{f})\right)} \mathrm{d} \boldsymbol{r}
\end{aligned}
$$

From equation A.11, we can straightforward derive the number of photoevents:

$$
\bar{K}=I(\mathbf{0})=\Sigma_{P} N\left(t_{1}+t_{2}\right)
$$

which is turbulence independent (neglecting scintillation). $\Sigma_{P}$ is the collecting area of a single telescope:

$$
\Sigma_{P}=\int\left[P_{1}(\boldsymbol{r})\right]^{2} \mathrm{~d} \boldsymbol{r}=\int\left[P_{2}(\boldsymbol{r})\right]^{2} \mathrm{~d} \boldsymbol{r}
$$

assuming for sake of simplicity that both pupils are identical.

Eq. A.11 also shows that multimode interferometry with image plane recombination continuously transmits the whole spatial frequencies $\boldsymbol{f}$. The complex visibility $V_{12}=V\left(\boldsymbol{f}_{12}\right)$ is then derived from the estimation of the coherent flux at the baseline frequency of the interferometer $\boldsymbol{f}_{12}$, that is:

$$
F_{12}^{c}=N \sqrt{t_{1} t_{2}} V_{12} S_{12}\left(\boldsymbol{f}_{12}\right)
$$

and the normalized spectrum of the interferogram takes the form:

$$
i\left(\boldsymbol{f}_{12}\right)=\frac{\sqrt{t_{1} t_{2}} V_{12}}{\left(t_{1}+t_{2}\right) \Sigma_{P}} S_{12}\left(\boldsymbol{f}_{12}\right)=\frac{\sqrt{t_{1} t_{2}} V_{12}}{\left(t_{1}+t_{2}\right)} T_{12}\left(\boldsymbol{f}_{12}\right)
$$

where $T_{12}(\boldsymbol{f})=S_{12}(\boldsymbol{f}) / \Sigma_{P}$ is the normalized interferometric transfer function, such as $\left|T_{12}\left(\boldsymbol{f}_{12}\right)\right|=1$. As a consequence, by analogy with the definition of the Strehl ratio for a single telescope, $\left|T_{12}\left(\boldsymbol{f}_{12}\right)\right|^{2}$ can be seen as the instantaneous multimode interferometric Strehl ratio. 


\section{A.3.2. Pupil plane recombination}

In such a scheme, fringes are formed in the pupil plane by means of geometrical operations of the entrance pupil (Chelli \& Mariotti 1986). This formalism is usually well suited to describe co-axial recombination with temporal coding where the pupils are superimposed at the beam splitter, hence the fringes formed in the pupil plane, as in the IOTA instrument. In practice, the fringes are form by translating one pupil over the other (translation of vector $\boldsymbol{f}_{12}$ ), and by introducing an optical path delay $\left(\theta_{1}(t)\right.$ and $\left.\theta_{2}(t)\right)$ on each beam (see e.g. Buscher 1988, Appendix A2) to modulate the fringe pattern. In this case, the complex amplitude of the superimposed electric fields writes:

$$
E(\boldsymbol{r})=\sqrt{t_{1}} P_{1}(\boldsymbol{r}) \exp ^{i \phi_{1}^{r}(\boldsymbol{r})} \Psi(\boldsymbol{r}) \exp ^{i \theta_{1}(t)}+\sqrt{t_{2}} P_{2}\left(\boldsymbol{r}+\lambda \boldsymbol{f}_{12}\right) \exp ^{i \phi_{2}^{r}\left(\boldsymbol{r}+\lambda \boldsymbol{f}_{12}\right)} \Psi\left(\boldsymbol{r}+\lambda \boldsymbol{f}_{12}\right) \exp ^{i \theta_{2}(t)}
$$

The fringe pattern $I(t)$ is then formed by focusing the light on one single pixel of the detector. Again, by rules of image formation, it writes as following:

$$
I(t)=\int|E(\boldsymbol{r})|^{2} \mathrm{~d} \boldsymbol{r}
$$

which, by making use of the expression of $E(\boldsymbol{r})$ rewrites:

$$
\begin{aligned}
I(t) & =\Sigma_{P} N\left(t_{1}+t_{2}\right)+N \sqrt{t_{1} t_{2}} \exp ^{i \Delta^{12} \theta(t)} \int P_{1}(\boldsymbol{r}) P_{2}\left(\boldsymbol{r}+\lambda \boldsymbol{f}_{12}\right) \exp ^{i\left(\phi_{1}^{r}(\boldsymbol{r})-\phi_{2}^{r}\left(\boldsymbol{r}+\lambda \boldsymbol{f}_{12}\right)\right)} \Psi(\boldsymbol{r}) \Psi^{*}\left(\boldsymbol{r}+\lambda \boldsymbol{f}_{12}\right) \mathrm{d} \boldsymbol{r}+C C \\
& =\Sigma_{P} N\left(t_{1}+t_{2}\right)+N \sqrt{t_{1} t_{2}} V\left(\boldsymbol{f}_{12}\right) S_{12}\left(\boldsymbol{f}_{12}\right) \exp ^{i \Delta^{12} \theta(t)}+N \sqrt{t_{1} t_{2}} V\left(-\boldsymbol{f}_{12}\right) S_{12}^{*}\left(-\boldsymbol{f}_{12}\right) \exp ^{-i \Delta^{12} \theta(t)}
\end{aligned}
$$

where $\Delta^{12} \theta(t)=\theta_{1}(t)-\theta_{2}(t)$ is the phase delay between the two beams which samples the fringe pattern. In the Fourier plane, the interferometric equation takes the form:

$$
I(\nu)=\Sigma_{P} N\left(t_{1}+t_{2}\right)+N \sqrt{t_{1} t_{2}} V\left(\boldsymbol{f}_{12}\right) S_{12}\left(\boldsymbol{f}_{12}\right) \delta_{\nu_{12}}+N \sqrt{t_{1} t_{2}} V^{*}\left(\boldsymbol{f}_{12}\right) S_{12}^{*}\left(\boldsymbol{f}_{12}\right) \delta_{-\nu_{12}}
$$

$\delta_{\nu}$ is the Dirac function, and $\nu_{12}$ is the temporal frequency at which the interferogram is modulated, that is the frequency of the moving piezoelectric mirror.

Note that, at the difference of the image plane recombination, pupil plane interferometry transmits only the frequency baseline $\boldsymbol{f}_{12}$. Nonetheless, the coherent flux, estimated in the Fourier plane at the frequency mirror $\left(\nu=\nu_{12}\right)$ takes the same form as in the image plane case, namely:

$$
F_{12}^{c}=N \sqrt{t_{1} t_{2}} V_{12} S_{12}\left(\boldsymbol{f}_{12}\right)
$$

and similarly the total number of detected photoevents is:

$$
\bar{K}=I(\mathbf{0})=\Sigma_{P} N\left(t_{1}+t_{2}\right)
$$

and the normalized spectrum of the interferogram at the frequency $\nu_{12}$ is:

$$
i\left(\nu_{12}\right)=\frac{\sqrt{t_{1} t_{2}} V_{12}}{\left(t_{1}+t_{2}\right) \Sigma_{P}} S_{12}\left(\boldsymbol{f}_{12}\right)
$$

\section{A.3.3. The interferometric phase error}

In previous sections, we have shown that independently of the chosen recombination scheme, the total flux, the coherent flux and consequently the normalized spectrum of the interferogram can take the form of Eqs. A.14, A.16, A.17, respectively. Using these expressions, Eqs. A.6. A.7. A.8 can be rewritten as following:

$$
\begin{aligned}
\mathrm{E}\left(|Q|^{2}\right) & =N^{2} t_{1} t_{2}\left|V_{12}\right|^{2}<\left|S_{12}\left(\boldsymbol{f}_{12}\right)\right|^{2}>_{\Phi}+\Sigma_{P} N\left(t_{1}+t_{2}\right)+N_{\text {pix }} \sigma_{\text {det }}^{2} \\
\mathrm{E}\left(Q^{2}\right) & =N^{2} t_{1} t_{2} V_{12}^{2}<S_{12}^{2}\left(\boldsymbol{f}_{12}\right)>_{\Phi} \\
\mathrm{E}(Q) & =N \sqrt{t_{1} t_{2}} V_{12}<S_{12}\left(\boldsymbol{f}_{12}\right)>_{\phi}
\end{aligned}
$$

NB: Chelli (1989) has shown that the error of the phase does not depends on the object phase, hence one can consider in the following that the object is centro-symmetric, that is $V_{12}=\left|V_{12}\right|=\operatorname{Re}\left[V_{12}\right]$.

It remains to derive in previous equations the moments of the interferometric transfer function $S_{12}\left(\boldsymbol{f}_{12}\right)$ :

$$
\begin{aligned}
<S_{12}\left(\boldsymbol{f}_{12}\right)>_{\Phi} & =\int P_{1}(\boldsymbol{r}) P_{2}\left(\boldsymbol{r}+\lambda \boldsymbol{f}_{12}\right)<\exp ^{i\left(\phi_{1}^{r}(\boldsymbol{r})-\phi_{2}^{r}\left(\boldsymbol{r}+\lambda \boldsymbol{f}_{12}\right)\right)}>_{\Phi} \mathrm{d} \boldsymbol{r} \\
& =\int P_{1}(\boldsymbol{r}) P_{2}\left(\boldsymbol{r}+\lambda \boldsymbol{f}_{12}\right) \exp ^{-\frac{1}{2}<\left|\left(\phi_{1}^{r}(\boldsymbol{r})-\phi_{2}^{r}\left(\boldsymbol{r}+\lambda \boldsymbol{f}_{12}\right)\right)\right|^{2}>_{\Phi}} \mathrm{d} \boldsymbol{r} \\
& =\Sigma_{P} \exp ^{-\sigma_{\phi_{r}}^{2}}
\end{aligned}
$$


E. Tatulli et al.: Multimode vs. single-mode interferometric phase, Online Material $p 4$

$$
\begin{aligned}
& <S_{12}^{2}\left(\boldsymbol{f}_{12}\right)>_{\Phi}=\int P_{1}(\boldsymbol{r}) P_{2}\left(\boldsymbol{r}+\lambda \boldsymbol{f}_{12}\right) P_{1}\left(\boldsymbol{r}^{\prime}\right) P_{2}\left(\boldsymbol{r}^{\prime}+\lambda \boldsymbol{f}_{12}\right)<\exp ^{i\left(\phi_{1}^{r}(\boldsymbol{r})-\phi_{2}^{r}\left(\boldsymbol{r}+\lambda \boldsymbol{f}_{12}\right)+\phi_{1}^{r}\left(\boldsymbol{r}^{\prime}\right)-\phi_{2}^{r}\left(\boldsymbol{r}^{\prime}+\lambda \boldsymbol{f}_{12}\right)\right)}>_{\Phi} \mathrm{d} \boldsymbol{r} \mathrm{d} \boldsymbol{r}^{\prime} \\
& =P_{1}(\boldsymbol{r}) P_{2}\left(\boldsymbol{r}+\lambda \boldsymbol{f}_{12}\right) P_{1}\left(\boldsymbol{r}^{\prime}\right) P_{2}\left(\boldsymbol{r}^{\prime}+\lambda \boldsymbol{f}_{12}\right) \exp ^{-\frac{1}{2}<\left|\left(\phi_{1}^{r}(\boldsymbol{r})-\phi_{2}^{r}\left(\boldsymbol{r}+\lambda \boldsymbol{f}_{12}\right)+\phi_{1}^{r}\left(\boldsymbol{r}^{\prime}\right)-\phi_{2}^{r}\left(\boldsymbol{r}^{\prime}+\lambda \boldsymbol{f}_{12}\right)\right)\right|^{2}>_{\Phi}} \mathrm{d} \boldsymbol{r} \mathrm{d} \boldsymbol{r}^{\prime} \\
& \text { knowing that }<\phi_{1}^{r}(\boldsymbol{r}) \phi_{1}^{r}\left(\boldsymbol{r}^{\prime}\right)>_{\Phi}=\sigma_{\phi_{r}}^{2}-\frac{1}{2} \mathcal{D}_{\phi_{r}}\left(\boldsymbol{r}, \boldsymbol{r}^{\prime}\right) \text {, it comes, } \\
& =\exp ^{-4 \sigma_{\phi_{r}}^{2}} \int P_{1}(\boldsymbol{r}) P_{2}\left(\boldsymbol{r}+\lambda \boldsymbol{f}_{12}\right) P_{1}\left(\boldsymbol{r}^{\prime}\right) P_{2}\left(\boldsymbol{r}^{\prime}+\lambda \boldsymbol{f}_{12}\right) \exp ^{\frac{1}{2} \mathcal{D}_{\phi_{r}}\left(\boldsymbol{r}, \boldsymbol{r}^{\prime}\right)+\frac{1}{2} \mathcal{D}_{\phi_{r}}\left(\boldsymbol{r}+\lambda \boldsymbol{f}_{12}, \boldsymbol{r}^{\prime}+\lambda \boldsymbol{f}_{12}\right)} \mathrm{d} \boldsymbol{r} \mathrm{d} \boldsymbol{r}^{\prime} \\
& \text { changing axes reference: } P_{1}(\boldsymbol{r}), P_{2}(\boldsymbol{r}) \rightarrow P(\boldsymbol{r}) \text { centered on } 0 \text {, } \\
& =\exp ^{-4 \sigma_{\phi_{r}}^{2}} \int\left[P(\boldsymbol{r}) P\left(\boldsymbol{r}^{\prime}\right) \exp ^{\frac{1}{2} \mathcal{D}_{\phi_{r}}\left(\boldsymbol{r}, \boldsymbol{r}^{\prime}\right)}\right]\left[P(\boldsymbol{r}) P\left(\boldsymbol{r}^{\prime}\right) \exp ^{\frac{1}{2} \mathcal{D}_{\phi_{r}}\left(\boldsymbol{r}, \boldsymbol{r}^{\prime}\right)}\right] \mathrm{d} \boldsymbol{r} \mathrm{d} \boldsymbol{r}^{\prime} \\
& <\left|S_{12}\left(\boldsymbol{f}_{12}\right)\right|^{2}>_{\Phi}=\int P_{1}(\boldsymbol{r}) P_{2}\left(\boldsymbol{r}+\lambda \boldsymbol{f}_{12}\right) P_{1}\left(\boldsymbol{r}^{\prime}\right) P_{2}\left(\boldsymbol{r}^{\prime}+\lambda \boldsymbol{f}_{12}\right)<\exp ^{i\left(\phi_{1}^{r}(\boldsymbol{r})-\phi_{2}^{r}\left(\boldsymbol{r}+\lambda \boldsymbol{f}_{12}\right)-\phi_{1}^{r}\left(\boldsymbol{r}^{\prime}\right)+\phi_{2}^{r}\left(\boldsymbol{r}^{\prime}+\lambda \boldsymbol{f}_{12}\right)\right)}>_{\Phi} \mathrm{d} \boldsymbol{r} \mathrm{d} \boldsymbol{r}^{\prime} \\
& =\int P_{1}(\boldsymbol{r}) P_{2}\left(\boldsymbol{r}+\lambda \boldsymbol{f}_{12}\right) P_{1}\left(\boldsymbol{r}^{\prime}\right) P_{2}\left(\boldsymbol{r}^{\prime}+\lambda \boldsymbol{f}_{12}\right) \exp ^{-\frac{1}{2}<\left|\left(\phi_{1}^{r}(\boldsymbol{r})-\phi_{2}^{r}\left(\boldsymbol{r}+\lambda \boldsymbol{f}_{12}\right)-\phi_{1}^{r}\left(\boldsymbol{r}^{\prime}\right)+\phi_{2}^{r}\left(\boldsymbol{r}^{\prime}+\lambda \boldsymbol{f}_{12}\right)\right)\right|^{2}>_{\Phi}} \mathrm{d} \boldsymbol{r} \mathrm{d} \boldsymbol{r}^{\prime} \\
& =\int P_{1}(\boldsymbol{r}) P_{2}\left(\boldsymbol{r}+\lambda \boldsymbol{f}_{12}\right) P_{1}\left(\boldsymbol{r}^{\prime}\right) P_{2}\left(\boldsymbol{r}^{\prime}+\lambda \boldsymbol{f}_{12}\right) \exp ^{-\frac{1}{2} \mathcal{D}_{\phi_{r}}\left(\boldsymbol{r}, \boldsymbol{r}^{\prime}\right)-\frac{1}{2} \mathcal{D}_{\phi_{r}}\left(\boldsymbol{r}+\lambda \boldsymbol{f}_{12}, \boldsymbol{r}^{\prime}+\lambda \boldsymbol{f}_{12}\right)} \mathrm{d} \boldsymbol{r} \mathrm{d} \boldsymbol{r}^{\prime} \\
& \text { changing axes reference: } \\
& =\int\left[P(\boldsymbol{r}) P\left(\boldsymbol{r}^{\prime}\right) \exp ^{-\frac{1}{2} \mathcal{D}_{\phi_{r}}\left(\boldsymbol{r}, \boldsymbol{r}^{\prime}\right)}\right]\left[P(\boldsymbol{r}) P\left(\boldsymbol{r}^{\prime}\right) \exp ^{-\frac{1}{2} \mathcal{D}_{\phi_{r}}\left(\boldsymbol{r}, \boldsymbol{r}^{\prime}\right)}\right] \mathrm{d} \boldsymbol{r} \mathrm{d} \boldsymbol{r}^{\prime}
\end{aligned}
$$

Putting Eqs. A.28 A.29, A.30 into Eqs. A.25, A.26, A.27, we finally find that the variance of the phase can be written as the quadratic sum of 3 terms, corresponding respectively to the detector, photon and atmospheric regimes $\sigma_{\phi}^{2}=$ $\sigma_{\operatorname{det}_{\phi}}^{2}+\sigma_{\text {phot }}^{2}+\sigma_{a t m_{\phi}}^{2}$, with:

$$
\begin{aligned}
\sigma_{\text {det }_{\phi}}^{2} & =\frac{1}{2} \frac{N_{\text {pix }} \sigma_{d e t}^{2}}{\Sigma_{P}^{2} N^{2} t_{1} t_{2}\left|V_{12}\right|^{2} \mathrm{e}^{-2 \sigma_{\phi_{r}}^{2}}} \\
\sigma_{\text {phot }}^{2} & =\frac{1}{2} \frac{t_{1}+t_{2}}{\Sigma_{P} N t_{1} t_{2}\left|V_{12}\right|^{2} \mathrm{e}^{-2 \sigma_{\phi_{r}}^{2}}} \\
\sigma_{\text {atm }}^{2} & =\frac{1}{2} \frac{\int\left[P(\boldsymbol{r}) P\left(\boldsymbol{r}^{\prime}\right) \mathrm{e}^{-\frac{1}{2} \mathcal{D}_{\phi_{r}}\left(\boldsymbol{r}, \boldsymbol{r}^{\prime}\right)}\right]^{2} \mathrm{~d} \boldsymbol{r} \mathrm{d} \boldsymbol{r}^{\prime}-\mathrm{e}^{-4 \sigma_{\phi_{r}}^{2}} \int\left[P(\boldsymbol{r}) P\left(\boldsymbol{r}^{\prime}\right) \mathrm{e}^{\left.\frac{1}{2} \mathcal{D}_{\phi_{r}}\left(\boldsymbol{r}, \boldsymbol{r}^{\prime}\right)\right]^{2} \mathrm{~d} \boldsymbol{r} \mathrm{d} \boldsymbol{r}^{\prime}}\right.}{\left[\int P^{2}(\boldsymbol{r}) \mathrm{d} \boldsymbol{r}\right]^{2} \mathrm{e}^{-2 \sigma_{\phi_{r}}^{2}}}
\end{aligned}
$$

We note that the expression of the variance in the photon noise case $\left(\sigma_{\text {phot }}^{2}\right)$ is the generalization of the formula found by Vannier et al. (2006) (Eq. (14)), when the visibility is corrupted by the partially AO-corrected turbulent phase.

\section{A.4. Phase noise in single-mode interferometry}

Because of the spatial filtering specific properties of single-mode devices, the single-mode interferometric equation changes slightly with respect to that of the multimode as we have to take into account the coupling efficiency which extenuates the number of coherent and incoherent photoevents. The coupling coefficients, respectively for the photometric $\left(\rho_{1}(V)\right.$, $\left.\rho_{2}(V)\right)$ and interferometric $\left(\rho^{12}(V)\right)$ channels write (Mège et al. 2003; Tatulli et al. 2004):

$$
\begin{aligned}
& \rho_{i}(V)=\rho_{0} \frac{\left(V * S_{i}\right)(\mathbf{0})}{\int S_{i}^{0}(\boldsymbol{f}) \mathrm{d} \boldsymbol{f}}, \quad(\mathrm{i}=[1,2]) \\
& =\rho_{0} \frac{\int V(f) P_{i}(\boldsymbol{r}) P_{i}(\boldsymbol{r}+\lambda \boldsymbol{f}) \exp ^{i\left(\phi_{i}^{r}(\boldsymbol{r})-\phi_{i}^{r}(\boldsymbol{r}+\lambda \boldsymbol{f})\right)} \mathrm{d} \boldsymbol{r} \mathrm{d} \boldsymbol{f}}{\left[\int P_{i}(\boldsymbol{r}) \mathrm{d} \boldsymbol{r}\right]^{2}} \\
& \rho^{12}(V)=\rho_{0} \frac{\left(V * S_{12}\right)\left(\boldsymbol{f}_{12}\right)}{\sqrt{\int S_{1}^{0}(\boldsymbol{f}) \mathrm{d} \boldsymbol{f} \int S_{2}^{0}(\boldsymbol{f}) \mathrm{d} \boldsymbol{f}}} \\
& =\rho_{0} \frac{\int V\left(f-\boldsymbol{f}_{12}\right) P_{1}(\boldsymbol{r}) P_{2}(\boldsymbol{r}+\lambda \boldsymbol{f}) \exp ^{i\left(\phi_{1}^{r}(\boldsymbol{r})-\phi_{2}^{r}(\boldsymbol{r}+\lambda \boldsymbol{f})\right)} \mathrm{d} \boldsymbol{r} \mathrm{d} \boldsymbol{f}}{\int P_{1}(\boldsymbol{r}) \mathrm{d} \boldsymbol{r} \int P_{2}(\boldsymbol{r}) \mathrm{d} \boldsymbol{r}}
\end{aligned}
$$

where $S_{1}^{0}(\boldsymbol{f}), S_{2}^{0}(\boldsymbol{f})$ are the photometric transfer functions of a perfect (atmosphere-free) interferometer, and $\rho_{0}$ the maximum coupling efficiency, shown to be $\sim 80 \%$ (Shaklan \& Roddier 1988).

And the single-mode interferometric equation writes:

$$
I(\boldsymbol{f})=\Sigma_{P} N t_{1} \rho_{1}(V) H_{1}(\boldsymbol{f})+\Sigma_{P} N t_{2} \rho_{2}(V) H_{2}(\boldsymbol{f})+\Sigma_{P} N \sqrt{t_{1} t_{2}} \rho^{12}(V) H_{12}(\boldsymbol{f})+\Sigma_{P} N \sqrt{t_{1} t_{2}}\left(\rho^{12}(V)\right)^{*} H_{12}^{*}(-\boldsymbol{f})
$$


E. Tatulli et al.: Multimode vs. single-mode interferometric phase, Online Material $p 5$

where $H_{1}(\boldsymbol{f}), H_{2}(\boldsymbol{f})$ and $H_{12}(\boldsymbol{f})$ are the normalized (i.e. $H_{1}(\mathbf{0})=H_{2}(\mathbf{0})=1$, and $H_{12}\left(\boldsymbol{f}_{12}\right)=1$ ) single-mode photometric and interferometric transfer functions, so-called the carrying wave (Mège 2002) which are fixed by the geometry of the single-mode device and therefore independent of the atmosphere. As a result, the single-mode coherent flux is defined by:

$$
F_{12}^{c}=\Sigma_{P} N \sqrt{t_{1} t_{2}} \rho^{12}(V)
$$

\section{A.4.1. Compact sources case}

In the case of compact sources - that is unresolved by the telescope, which is the most common case in interferometry Equations A.34 and A.35 are taking the simplified following form:

$$
\begin{aligned}
\rho_{i}(V) & =\rho_{0} \frac{\int P_{i}(\boldsymbol{r}) P_{i}(\boldsymbol{r}+\lambda \boldsymbol{f}) \exp ^{i\left(\phi_{i}^{r}(\boldsymbol{r})-\phi_{i}^{r}(\boldsymbol{r}+\lambda \boldsymbol{f})\right)} \mathrm{d} \boldsymbol{r} \mathrm{f} \boldsymbol{f}}{\left[\int P_{i}(\boldsymbol{r}) \mathrm{d} \boldsymbol{r}\right]^{2}}=\rho_{0} \mathcal{S}_{i} \\
\rho^{12}(V) & =\rho_{0} V_{12} \frac{\int P_{1}(\boldsymbol{r}) P_{2}(\boldsymbol{r}+\lambda \boldsymbol{f}) \exp ^{i\left(\phi_{1}^{r}(\boldsymbol{r})-\phi_{2}^{r}(\boldsymbol{r}+\lambda \boldsymbol{f})\right)} \mathrm{d} \boldsymbol{r} \mathrm{d} \boldsymbol{f}}{\int P_{1}(\boldsymbol{r}) \mathrm{d} \boldsymbol{r} \int P_{2}(\boldsymbol{r}) \mathrm{d} \boldsymbol{r}}=\rho_{0} V_{12} \rho_{12}
\end{aligned}
$$

where $\mathcal{S}_{i}$ is by definition the instantaneous Strehl ratio relative to the $i^{\text {th }}$ telescope, and with:

$\rho_{12}=\frac{\int P_{1}(\boldsymbol{r}) P_{2}(\boldsymbol{r}+\lambda \boldsymbol{f}) \exp ^{i\left(\phi_{1}^{r}(\boldsymbol{r})-\phi_{2}^{r}(\boldsymbol{r}+\lambda \boldsymbol{f})\right)} \mathrm{d} \boldsymbol{r} \mathrm{d} \boldsymbol{f}}{\int P_{1}(\boldsymbol{r}) \mathrm{d} \boldsymbol{r} \int P_{2}(\boldsymbol{r}) \mathrm{d} \boldsymbol{r}}$

$\left|\rho_{12}\right|^{2}$ being by analogy the instantaneous single-mode interferometric Strehl ratio. The interferometric equation finally rewrites:

$$
I(\boldsymbol{f})=\Sigma_{P} N t_{1} \rho_{0} \mathcal{S}_{1} H_{1}(\boldsymbol{f})+\Sigma_{P} N t_{2} \rho_{0} \mathcal{S}_{2} H_{2}(\boldsymbol{f})+\Sigma_{P} N \sqrt{t_{1} t_{2}} V_{12} \rho_{12} H_{12}(\boldsymbol{f})+\Sigma_{P} N \sqrt{t_{1} t_{2}} \rho_{0} V_{12}^{*} \rho_{12}^{*} H_{12}^{*}(-\boldsymbol{f})
$$

And the single-mode coherent flux, at the spatial frequency $\boldsymbol{f}_{12}$ takes the form:

$$
F_{12}^{c}=\Sigma_{P} N \sqrt{t_{1} t_{2}} V_{12} \rho_{12}
$$

whereas the number of photoevents writes:

$$
\bar{K}=I(\mathbf{0})=\Sigma_{P} N \rho_{0} \mathcal{S}_{1}+\Sigma_{P} N t_{2} \rho_{0} \mathcal{S}_{2}
$$

which depends on the turbulent atmosphere through the fluctuations of the instantaneous Strehl ratio $\mathcal{S}_{1}$ and $\mathcal{S}_{2}$. And the normalized spectrum of the interferogram is:

$$
i\left(\boldsymbol{f}_{12}\right)=\frac{\sqrt{t_{1} t_{2}} \rho_{12} V_{12}}{t_{1} \mathcal{S}_{1}+t_{2} \mathcal{S}_{2}}
$$

The generic equations of the phase noise thus rewrites:

$$
\begin{aligned}
\mathrm{E}\left(|Q|^{2}\right) & =\Sigma_{P}^{2} N^{2} t_{1} t_{2} \rho_{0}^{2}\left|V_{12}\right|^{2}<\left|\rho_{12}\right|^{2}>_{\Phi}+\Sigma_{P} N t_{1} \rho_{0}<\mathcal{S}_{1}>_{\Phi}+\Sigma_{P} N t_{2} \rho_{0}<\mathcal{S}_{2}>_{\Phi}+N_{\text {pix }} \sigma_{\text {det }}^{2} \\
\mathrm{E}\left(Q^{2}\right) & =\Sigma_{P}^{2} N^{2} t_{1} t_{2} \rho_{0}^{2} V_{12}^{2}<\rho_{12}^{2}>_{\Phi} \\
\mathrm{E}(Q) & =\Sigma_{P} N \sqrt{t_{1} t_{2}} \rho_{0} V_{12}<\rho_{12}>_{\Phi}
\end{aligned}
$$

It now remains to compute the moments of the coupling coefficients:

$<\mathcal{S}_{i}>_{\Phi}=\overline{\mathcal{S}_{i}}, \quad(\mathrm{i}=[1,2])$

where $\overline{\mathcal{S}_{i}}$ is the long exposure Strehl ratio of the $i^{\text {th }}$ telescope. Assuming equivalent AO correction for both telescopes, we have $\overline{\mathcal{S}_{1}}=\overline{\mathcal{S}_{2}}=\overline{\mathcal{S}}$

$$
<\rho_{12}>_{\Phi}=\frac{\int P_{1}(\boldsymbol{r}) P_{2}(\boldsymbol{r}+\lambda \boldsymbol{f})<\exp ^{i\left(\phi_{1}^{r}(\boldsymbol{r})-\phi_{2}^{r}(\boldsymbol{r}+\lambda \boldsymbol{f})\right)}>_{\Phi} \mathrm{d} \boldsymbol{r} \mathrm{d} \boldsymbol{f}}{\int P_{1}(\boldsymbol{r}) \mathrm{d} \boldsymbol{r} \int P_{2}(\boldsymbol{r}) \mathrm{d} \boldsymbol{r}}=\mathrm{e}^{-\sigma_{\phi_{r}}^{2}} \frac{\int P_{1}(\boldsymbol{r}) P_{2}(\boldsymbol{r}+\lambda \boldsymbol{f}) \mathrm{d} \boldsymbol{r} \mathrm{d} \boldsymbol{f}}{\int P_{1}(\boldsymbol{r}) \mathrm{d} \boldsymbol{r} \int P_{2}(\boldsymbol{r}) \mathrm{d} \boldsymbol{r}}=\mathrm{e}^{-\sigma_{\phi_{r}}^{2}}
$$

which shows that the long exposure interferometric Strehl ratio is equal to the coherent energy $\mathrm{e}^{-\sigma_{\phi_{r}}^{2}}$.

$$
\begin{aligned}
& <\rho_{12}^{2}>_{\Phi}=\frac{\int P_{1}(\boldsymbol{r}) P_{2}(\boldsymbol{r}+\lambda \boldsymbol{f}) P_{1}\left(\boldsymbol{r}^{\prime}\right) P_{2}\left(\boldsymbol{r}^{\prime}+\lambda \boldsymbol{f}^{\prime}\right)<\exp ^{i\left(\phi_{1}^{r}(\boldsymbol{r})+\phi_{1}^{r}\left(\boldsymbol{r}^{\prime}\right)-\phi_{2}^{r}(\boldsymbol{r}+\lambda \boldsymbol{f})-\phi_{2}^{r}\left(\boldsymbol{r}^{\prime}+\lambda \boldsymbol{f}^{\prime}\right)\right)}>_{\Phi} \mathrm{d} \boldsymbol{r} \mathrm{d} \boldsymbol{r}^{\prime} \mathrm{d} \boldsymbol{f} \mathrm{d} \boldsymbol{f}^{\prime}}{\left[\int P_{1}(\boldsymbol{r}) \mathrm{d} \boldsymbol{r} \int P_{2}(\boldsymbol{r}) \mathrm{d} \boldsymbol{r}\right]^{2}} \\
& =\mathrm{e}^{-4 \sigma_{\phi_{r}}^{2}} \frac{\int P_{1}(\boldsymbol{r}) P_{2}(\boldsymbol{r}+\lambda \boldsymbol{f}) P_{1}\left(\boldsymbol{r}^{\prime}\right) P_{2}\left(\boldsymbol{r}^{\prime}+\lambda \boldsymbol{f}^{\prime}\right) \exp ^{\frac{1}{2} \mathcal{D}_{\phi_{r}}\left(\boldsymbol{r}, \boldsymbol{r}^{\prime}\right)+\frac{1}{2} \mathcal{D}_{\phi_{r}}\left(\boldsymbol{r}+\lambda \boldsymbol{f}, \boldsymbol{r}^{\prime}+\lambda \boldsymbol{f}^{\prime}\right)} \mathrm{d} \boldsymbol{r} \mathrm{d} \boldsymbol{r}^{\prime} \mathrm{d} \boldsymbol{f} \mathrm{d} \boldsymbol{f}^{\prime}}{\left[\int P_{1}(\boldsymbol{r}) \mathrm{d} \boldsymbol{r} \int P_{2}(\boldsymbol{r}) \mathrm{d} \boldsymbol{r}\right]^{2}} \\
& \text { change of variables } \boldsymbol{r}+\lambda \boldsymbol{f} \rightarrow \boldsymbol{u}, \boldsymbol{r}^{\prime}+\lambda \boldsymbol{f}^{\prime} \rightarrow \boldsymbol{u}^{\prime} \text { : } \\
& =\mathrm{e}^{-4 \sigma_{\phi_{r}}^{2}} \frac{\int P_{1}(\boldsymbol{r}) P_{1}\left(\boldsymbol{r}^{\prime}\right) \exp ^{\frac{1}{2} \mathcal{D}_{\phi_{r}}\left(\boldsymbol{r}, \boldsymbol{r}^{\prime}\right)} \mathrm{d} \boldsymbol{r} \mathrm{d} \boldsymbol{r}^{\prime} \int P_{2}(\boldsymbol{r}) P_{2}\left(\boldsymbol{r}^{\prime}\right) \exp ^{\frac{1}{2} \mathcal{D}_{\phi_{r}}\left(\boldsymbol{r}, \boldsymbol{r}^{\prime}\right)} \mathrm{d} \boldsymbol{r} \mathrm{d} \boldsymbol{r}^{\prime}}{\left[\int P_{1}(\boldsymbol{r}) \mathrm{d} \boldsymbol{r} \int P_{2}(\boldsymbol{r}) \mathrm{d} \boldsymbol{r}\right]^{2}}
\end{aligned}
$$


E. Tatulli et al.: Multimode vs. single-mode interferometric phase, Online Material $p 6$

$$
\begin{aligned}
& <\left|\rho_{12}\right|^{2}>_{\Phi}=\frac{\int P_{1}(\boldsymbol{r}) P_{2}(\boldsymbol{r}+\lambda \boldsymbol{f}) P_{1}\left(\boldsymbol{r}^{\prime}\right) P_{2}\left(\boldsymbol{r}^{\prime}+\lambda \boldsymbol{f}^{\prime}\right)<\exp ^{i\left(\phi_{1}^{r}(\boldsymbol{r})-\phi_{1}^{r}\left(\boldsymbol{r}^{\prime}\right)-\phi_{2}^{r}(\boldsymbol{r}+\lambda \boldsymbol{f})+\phi_{2}^{r}\left(\boldsymbol{r}^{\prime}+\lambda \boldsymbol{f}^{\prime}\right)\right)}>_{\Phi} \mathrm{d} \boldsymbol{r} \mathrm{d} \boldsymbol{r}^{\prime} \mathrm{d} \boldsymbol{f} \mathrm{d} \boldsymbol{f}^{\prime}}{\left[\int P_{1}(\boldsymbol{r}) \mathrm{d} \boldsymbol{r} \int P_{2}(\boldsymbol{r}) \mathrm{d} \boldsymbol{r}\right]^{2}} \\
& =\frac{\int P_{1}(\boldsymbol{r}) P_{2}(\boldsymbol{r}+\lambda \boldsymbol{f}) P_{1}\left(\boldsymbol{r}^{\prime}\right) P_{2}\left(\boldsymbol{r}^{\prime}+\lambda \boldsymbol{f}^{\prime}\right) \exp ^{-\frac{1}{2} \mathcal{D}_{\phi_{r}}\left(\boldsymbol{r}, \boldsymbol{r}^{\prime}\right)-\frac{1}{2} \mathcal{D}_{\phi_{r}}\left(\boldsymbol{r}+\lambda \boldsymbol{f}, \boldsymbol{r}^{\prime}+\lambda \boldsymbol{f}^{\prime}\right)} \mathrm{d} \boldsymbol{r} \mathrm{d} \boldsymbol{r}^{\prime} \mathrm{d} \boldsymbol{f} \mathrm{d} \boldsymbol{f}^{\prime}}{\left[\int P_{1}(\boldsymbol{r}) \mathrm{d} \boldsymbol{r} \int P_{2}(\boldsymbol{r}) \mathrm{d} \boldsymbol{r}\right]^{2}} \\
& \text { change of variables } \boldsymbol{r}+\lambda \boldsymbol{f} \rightarrow \boldsymbol{u}, \boldsymbol{r}^{\prime}+\lambda \boldsymbol{f}^{\prime} \rightarrow \boldsymbol{u}^{\prime} \text { : } \\
& =\frac{\int P_{1}(\boldsymbol{r}) P_{1}\left(\boldsymbol{r}^{\prime}\right) \exp ^{-\frac{1}{2} \mathcal{D}_{\phi_{r}}\left(\boldsymbol{r}, \boldsymbol{r}^{\prime}\right)} \mathrm{d} \boldsymbol{r} \mathrm{d} \boldsymbol{r}^{\prime} \int P_{2}(\boldsymbol{r}) P_{2}\left(\boldsymbol{r}^{\prime}\right) \exp ^{-\frac{1}{2} \mathcal{D}_{\phi_{r}}\left(\boldsymbol{r}, \boldsymbol{r}^{\prime}\right)} \mathrm{d} \boldsymbol{r} \mathrm{d} \boldsymbol{r}^{\prime}}{\left[\int P_{1}(\boldsymbol{r}) \mathrm{d} \boldsymbol{r} \int P_{2}(\boldsymbol{r}) \mathrm{d} \boldsymbol{r}\right]^{2}}
\end{aligned}
$$

Putting Eqs. A.50 A.51, A.52 A.53 into Eqs. A.47, A.48, A.49, we find again that the variance of the phase can be written as the quadratic sum of 3 terms, corresponding respectively to the detector, photon and atmospheric regimes $\sigma_{\phi}^{2}=\sigma_{\operatorname{det}_{\phi}}^{2}+\sigma_{\text {phot }_{\phi}}^{2}+\sigma_{\text {atm }}^{2}$, with:

$$
\begin{aligned}
\sigma_{\text {det }}^{2} & =\frac{1}{2} \frac{N_{\text {pix }} \sigma_{\text {det }}^{2}}{\rho_{0}^{2} \Sigma_{P}^{2} N^{2} t_{1} t_{2}\left|V_{12}\right|^{2} \mathrm{e}^{-2 \sigma_{\phi_{r}}^{2}}} \\
\sigma_{\text {phot }}^{2} & =\frac{1}{2} \frac{\left(t_{1}+t_{2}\right) \overline{\mathcal{S}}}{\rho_{0} \Sigma_{P} N t_{1} t_{2}\left|V_{12}\right|^{2} \mathrm{e}^{-2 \sigma_{\phi_{r}}^{2}}} \\
\sigma_{\text {atm }}^{2} & =\frac{1}{2} \frac{\left[\int P(\boldsymbol{r}) P\left(\boldsymbol{r}^{\prime}\right) \mathrm{e}^{-\frac{1}{2} \mathcal{D}_{\phi_{r}}\left(\boldsymbol{r}, \boldsymbol{r}^{\prime}\right)} \mathrm{d} \boldsymbol{r} \mathrm{d} \boldsymbol{r}^{\prime}\right]^{2}-\mathrm{e}^{-4 \sigma_{\phi_{r}}^{2}}\left[\int P(\boldsymbol{r}) P\left(\boldsymbol{r}^{\prime}\right) \mathrm{e}^{\frac{1}{2} \mathcal{D}_{\phi_{r}}\left(\boldsymbol{r}, \boldsymbol{r}^{\prime}\right)} \mathrm{d} \boldsymbol{r} \mathrm{d} \boldsymbol{r}^{\prime}\right]^{2}}{\left[\int P(\boldsymbol{r}) \mathrm{d} \boldsymbol{r}\right]^{4} \mathrm{e}^{-2 \sigma_{\phi_{r}}^{2}}}
\end{aligned}
$$

\section{Appendix B: Coherent flux dropouts - Rician density probability}

By analogy with the study of Canales \& Cagigal (1999) on the statistics of the instantaneous Strehl ratio in partial AO correction, the instantaneous interferometric Strehl ratio, namely $I=\left|T_{12}\right|^{2}$ and $I=\left|\rho_{12}\right|^{2}$ for multimode and single-mode interferometers respectively are shown to follow Rician density probability statistics of the form:

$$
d p(I)=\frac{1}{2 \sigma^{2}} \exp \left(-\frac{I+a^{2}}{2 \sigma^{2}}\right) I_{0}\left(-\frac{a \sqrt{I}}{\sigma^{2}}\right)
$$

In this appendix, we aim to derive the theoretical expression of the moments of the Rician distribution (namely $a$ and $\sigma$ ), in both multimode and single-mode cases. Following Canales \& Cagigal (1999), the quantity $a$ and $\sigma$ can be expressed from the moments of the real $\left(A_{r}\right)$ and imaginary $\left(\overline{A_{i}}\right)$ parts of the complex instantaneous interferometric Strehl ratio (i.e. such as $\left.I=A_{r}^{2}+A_{i}^{2}\right)$ :

$$
a^{4}=<A_{r}>_{\Phi}^{4}+2<A_{r}>_{\Phi}^{2}\left(\sigma_{i}^{2}-\sigma_{r}^{2}\right)-\left(\sigma_{i}^{2}-\sigma_{r}^{2}\right)^{2}
$$

$2 \sigma^{2}=\sigma_{r}^{2}+\sigma_{i}^{2}+<A_{r}>_{\Phi}^{2}-a^{2}$

where $\sigma_{r}^{2}=<A_{r}^{2}>_{\Phi}-<A_{r}>_{\Phi}^{2}$ and $\sigma_{i}^{2}=<A_{i}^{2}>_{\Phi}-<A_{i}>_{\Phi}^{2}$.

\section{B.1. Multimode case}

In multimode interferometry we have $I=\left|T_{12}\right|^{2}$, hence:

$$
\begin{aligned}
& A_{r}=\operatorname{Re}\left(T_{12}\right)=\frac{\int P_{1}(\boldsymbol{r}) P_{2}(\boldsymbol{r}+\lambda \boldsymbol{f}) \cos \left(\phi_{1}^{r}(\boldsymbol{r})-\phi_{2}^{r}(\boldsymbol{r}+\lambda \boldsymbol{f})\right) \mathrm{d} \boldsymbol{r}}{\Sigma_{P}} \\
& A_{i}=\operatorname{Im}\left(T_{12}\right)=\frac{\int P_{1}(\boldsymbol{r}) P_{2}(\boldsymbol{r}+\lambda \boldsymbol{f}) \sin \left(\phi_{1}^{r}(\boldsymbol{r})-\phi_{2}^{r}(\boldsymbol{r}+\lambda \boldsymbol{f})\right) \mathrm{d} \boldsymbol{r}}{\Sigma_{P}}
\end{aligned}
$$

Following the same approach as in appendix A.3, we compute the first and second order moments of these quantities.

$$
\begin{aligned}
& <A_{r}>_{\Phi}=\operatorname{Re}\left(T_{12}\right)=\frac{\int P_{1}(\boldsymbol{r}) P_{2}(\boldsymbol{r}+\lambda \boldsymbol{f})<\cos \left(\phi_{1}^{r}(\boldsymbol{r})-\phi_{2}^{r}(\boldsymbol{r}+\lambda \boldsymbol{f})\right)>_{\Phi} \mathrm{d} \boldsymbol{r}}{\Sigma_{P}}=\exp ^{-\sigma_{\phi_{r}}^{2}} \\
& <A_{i}>_{\Phi}=\operatorname{Im}\left(T_{12}\right)=\frac{\int P_{1}(\boldsymbol{r}) P_{2}(\boldsymbol{r}+\lambda \boldsymbol{f})<\sin \left(\phi_{1}^{r}(\boldsymbol{r})-\phi_{2}^{r}(\boldsymbol{r}+\lambda \boldsymbol{f})\right)>_{\Phi} \mathrm{d} \boldsymbol{r}}{\Sigma_{P}}=0
\end{aligned}
$$


E. Tatulli et al.: Multimode vs. single-mode interferometric phase, Online Material $p$

$$
\begin{aligned}
& <A_{r}^{2}>_{\Phi}=\frac{\int P_{1}(\boldsymbol{r}) P_{2}\left(\boldsymbol{r}+\lambda \boldsymbol{f}_{12}\right) P_{1}\left(\boldsymbol{r}^{\prime}\right) P_{2}\left(\boldsymbol{r}^{\prime}+\lambda \boldsymbol{f}_{12}\right)<\cos \left(\phi_{1}^{r}(\boldsymbol{r})-\phi_{2}^{r}\left(\boldsymbol{r}+\lambda \boldsymbol{f}_{12}\right)\right) \cos \left(\phi_{1}^{r}\left(\boldsymbol{r}^{\prime}\right)-\phi_{2}^{r}\left(\boldsymbol{r}^{\prime}+\lambda \boldsymbol{f}_{12}\right)\right)>_{\Phi} \mathrm{d} \boldsymbol{r} \mathrm{d} \boldsymbol{r}^{\prime}}{\Sigma_{P}^{2}} \\
& \text { using } \cos (a) \cos (b)=(\cos (a-b)+\cos (a+b)) / 2 \\
& =\frac{1}{2} \frac{\int P_{1}(\boldsymbol{r}) P_{2}\left(\boldsymbol{r}+\lambda \boldsymbol{f}_{12}\right) P_{1}\left(\boldsymbol{r}^{\prime}\right) P_{2}\left(\boldsymbol{r}^{\prime}+\lambda \boldsymbol{f}_{12}\right)<\cos \left(\phi_{1}^{r}(\boldsymbol{r})-\phi_{2}^{r}\left(\boldsymbol{r}+\lambda \boldsymbol{f}_{12}\right)-\phi_{1}^{r}\left(\boldsymbol{r}^{\prime}\right)+\phi_{2}^{r}\left(\boldsymbol{r}^{\prime}+\lambda \boldsymbol{f}_{12}\right)\right)>_{\Phi} \mathrm{d} \boldsymbol{r} \mathrm{d} \boldsymbol{r}^{\prime}}{\Sigma_{P}^{2}} \\
& +\frac{1}{2} \frac{\int P_{1}(\boldsymbol{r}) P_{2}\left(\boldsymbol{r}+\lambda \boldsymbol{f}_{12}\right) P_{1}\left(\boldsymbol{r}^{\prime}\right) P_{2}\left(\boldsymbol{r}^{\prime}+\lambda \boldsymbol{f}_{12}\right)<\cos \left(\phi_{1}^{r}(\boldsymbol{r})-\phi_{2}^{r}\left(\boldsymbol{r}+\lambda \boldsymbol{f}_{12}\right)+\phi_{1}^{r}\left(\boldsymbol{r}^{\prime}\right)-\phi_{2}^{r}\left(\boldsymbol{r}^{\prime}+\lambda \boldsymbol{f}_{12}\right)\right)>_{\Phi} \mathrm{d} \boldsymbol{r} \mathrm{d} \boldsymbol{r}^{\prime}}{\Sigma_{P}^{2}} \\
& \text { changing axes reference: } P_{1}, P_{2} \text { centered on } 0 \text {, } \\
& =\frac{1}{2} \frac{\int\left[P(\boldsymbol{r}) P\left(\boldsymbol{r}^{\prime}\right) \exp ^{-\frac{1}{2} \mathcal{D}_{\phi_{r}}\left(\boldsymbol{r}, \boldsymbol{r}^{\prime}\right)}\right]\left[P(\boldsymbol{r}) P\left(\boldsymbol{r}^{\prime}\right) \exp ^{-\frac{1}{2} \mathcal{D}_{\phi_{r}}\left(\boldsymbol{r}, \boldsymbol{r}^{\prime}\right)}\right] \mathrm{d} \boldsymbol{r} \mathrm{d} \boldsymbol{r}^{\prime}}{\left[\int P^{2}(\boldsymbol{r}) \mathrm{d} \boldsymbol{r}\right]^{2}} \\
& +\frac{1}{2} \exp ^{-4 \sigma_{\phi_{r}}^{2}} \frac{\int\left[P(\boldsymbol{r}) P\left(\boldsymbol{r}^{\prime}\right) \exp ^{\frac{1}{2} \mathcal{D}_{\phi_{r}}\left(\boldsymbol{r}, \boldsymbol{r}^{\prime}\right)}\right]\left[P(\boldsymbol{r}) P\left(\boldsymbol{r}^{\prime}\right) \exp ^{\frac{1}{2} \mathcal{D}_{\phi_{r}}\left(\boldsymbol{r}, \boldsymbol{r}^{\prime}\right)}\right] \mathrm{d} \boldsymbol{r} \mathrm{d} \boldsymbol{r}^{\prime}}{\left[\int P^{2}(\boldsymbol{r}) \mathrm{d} \boldsymbol{r}\right]^{2}}
\end{aligned}
$$

$$
\begin{aligned}
<A_{i}^{2}>_{\Phi}= & \frac{\int P_{1}(\boldsymbol{r}) P_{2}\left(\boldsymbol{r}+\lambda \boldsymbol{f}_{12}\right) P_{1}\left(\boldsymbol{r}^{\prime}\right) P_{2}\left(\boldsymbol{r}^{\prime}+\lambda \boldsymbol{f}_{12}\right)<\sin \left(\phi_{1}^{r}(\boldsymbol{r})-\phi_{2}^{r}\left(\boldsymbol{r}+\lambda \boldsymbol{f}_{12}\right)\right) \sin \left(\phi_{1}^{r}\left(\boldsymbol{r}^{\prime}\right)-\phi_{2}^{r}\left(\boldsymbol{r}^{\prime}+\lambda \boldsymbol{f}_{12}\right)\right)>_{\Phi} \mathrm{d} \boldsymbol{r} \mathrm{d} \boldsymbol{r}^{\prime}}{\Sigma_{P}^{2}} \\
& \text { using } \sin (a) \sin (b)=(\cos (a-b)-\cos (a+b)) / 2 \\
= & \frac{1}{2} \frac{\int P_{1}(\boldsymbol{r}) P_{2}\left(\boldsymbol{r}+\lambda \boldsymbol{f}_{12}\right) P_{1}\left(\boldsymbol{r}^{\prime}\right) P_{2}\left(\boldsymbol{r}^{\prime}+\lambda \boldsymbol{f}_{12}\right)<\cos \left(\phi_{1}^{r}(\boldsymbol{r})-\phi_{2}^{r}\left(\boldsymbol{r}+\lambda \boldsymbol{f}_{12}\right)-\phi_{1}^{r}\left(\boldsymbol{r}^{\prime}\right)+\phi_{2}^{r}\left(\boldsymbol{r}^{\prime}+\lambda \boldsymbol{f}_{12}\right)\right)>_{\Phi} \mathrm{d} \boldsymbol{r} \mathrm{d} \boldsymbol{r}^{\prime}}{\Sigma_{P}^{2}} \\
& -\frac{1}{2} \frac{\int P_{1}(\boldsymbol{r}) P_{2}\left(\boldsymbol{r}+\lambda \boldsymbol{f}_{12}\right) P_{1}\left(\boldsymbol{r}^{\prime}\right) P_{2}\left(\boldsymbol{r}^{\prime}+\lambda \boldsymbol{f}_{12}\right)<\cos \left(\phi_{1}^{r}(\boldsymbol{r})-\phi_{2}^{r}\left(\boldsymbol{r}+\lambda \boldsymbol{f}_{12}\right)+\phi_{1}^{r}\left(\boldsymbol{r}^{\prime}\right)-\phi_{2}^{r}\left(\boldsymbol{r}^{\prime}+\lambda \boldsymbol{f}_{12}\right)\right)>_{\Phi} \mathrm{d} \boldsymbol{r} \mathrm{d} \boldsymbol{r}^{\prime}}{\Sigma_{P}^{2}}
\end{aligned}
$$

changing axes reference: $P_{1}, P_{2}$ centered on 0 ,

$$
\begin{aligned}
& =\frac{1}{2} \frac{\int\left[P(\boldsymbol{r}) P\left(\boldsymbol{r}^{\prime}\right) \exp ^{-\frac{1}{2} \mathcal{D}_{\phi_{r}}\left(\boldsymbol{r}, \boldsymbol{r}^{\prime}\right)}\right]\left[P(\boldsymbol{r}) P\left(\boldsymbol{r}^{\prime}\right) \exp ^{-\frac{1}{2} \mathcal{D}_{\phi_{r}}\left(\boldsymbol{r}, \boldsymbol{r}^{\prime}\right)}\right] \mathrm{d} \boldsymbol{r} \mathrm{d} \boldsymbol{r}^{\prime}}{\left[\int P^{2}(\boldsymbol{r}) \mathrm{d} \boldsymbol{r}\right]^{2}} \\
& -\frac{1}{2} \exp ^{-4 \sigma_{\phi_{r}}^{2}} \frac{\int\left[P(\boldsymbol{r}) P\left(\boldsymbol{r}^{\prime}\right) \exp ^{\frac{1}{2} \mathcal{D}_{\phi_{r}}\left(\boldsymbol{r}, \boldsymbol{r}^{\prime}\right)}\right]\left[P(\boldsymbol{r}) P\left(\boldsymbol{r}^{\prime}\right) \exp ^{\left.\frac{1}{2} \mathcal{D}_{\phi_{r}}\left(\boldsymbol{r}, \boldsymbol{r}^{\prime}\right)\right] \mathrm{d} \boldsymbol{r} \mathrm{d} \boldsymbol{r}^{\prime}}\right.}{\left[\int P^{2}(\boldsymbol{r}) \mathrm{d} \boldsymbol{r}\right]^{2}}
\end{aligned}
$$

Using Eqs. B.6. B.7. B.8 B.9 in Eqs. B.2, B.3, we obtain the theoretical expressions of the parameters $a$ and $\sigma$ of the Rician distribution is the multimode case, as summarized in Table B.1 of the appendix.

\section{B.2. Single-mode case}

In single-mode interferometry we have $I=\left|\rho_{12}\right|^{2}$, hence:

$$
\begin{aligned}
& A_{r}=\operatorname{Re}\left(\rho_{12}\right)=\frac{\int P_{1}(\boldsymbol{r}) P_{2}(\boldsymbol{r}+\lambda \boldsymbol{f}) \cos \left(\phi_{1}^{r}(\boldsymbol{r})-\phi_{2}^{r}(\boldsymbol{r}+\lambda \boldsymbol{f})\right) \mathrm{d} \boldsymbol{r} \mathrm{d} \boldsymbol{f}}{\int P_{1}(\boldsymbol{r}) \mathrm{d} \boldsymbol{r} \int P_{2}(\boldsymbol{r}) \mathrm{d} \boldsymbol{r}} \\
& A_{i}=\operatorname{Im}\left(\rho_{12}\right)=\frac{\int P_{1}(\boldsymbol{r}) P_{2}(\boldsymbol{r}+\lambda \boldsymbol{f}) \sin \left(\phi_{1}^{r}(\boldsymbol{r})-\phi_{2}^{r}(\boldsymbol{r}+\lambda \boldsymbol{f})\right) \mathrm{d} \boldsymbol{r} \mathrm{d} \boldsymbol{f}}{\int P_{1}(\boldsymbol{r}) \mathrm{d} \boldsymbol{r} \int P_{2}(\boldsymbol{r}) \mathrm{d} \boldsymbol{r}}
\end{aligned}
$$

Following the same formalism as previously, we compute the first and second order moments of these quantities.

$$
\begin{aligned}
& <A_{r}>_{\Phi}=\frac{\int P_{1}(\boldsymbol{r}) P_{2}(\boldsymbol{r}+\lambda \boldsymbol{f})<\cos \left(\phi_{1}^{r}(\boldsymbol{r})-\phi_{2}^{r}(\boldsymbol{r}+\lambda \boldsymbol{f})\right)>_{\Phi} \mathrm{d} \boldsymbol{r} \mathrm{d} \boldsymbol{f}}{\int P_{1}(\boldsymbol{r}) \mathrm{d} \boldsymbol{r} \int P_{2}(\boldsymbol{r}) \mathrm{d} \boldsymbol{r}}=\exp ^{-\sigma_{\phi_{r}}^{2}} \\
& <A_{i}>_{\Phi}=\frac{\int P_{1}(\boldsymbol{r}) P_{2}(\boldsymbol{r}+\lambda \boldsymbol{f})<\sin \left(\phi_{1}^{r}(\boldsymbol{r})-\phi_{2}^{r}(\boldsymbol{r}+\lambda \boldsymbol{f})\right)>_{\Phi} \mathrm{d} \boldsymbol{r} \mathrm{d} \boldsymbol{f}}{\int P_{1}(\boldsymbol{r}) \mathrm{d} \boldsymbol{r} \int P_{2}(\boldsymbol{r}) \mathrm{d} \boldsymbol{r}}=0
\end{aligned}
$$


E. Tatulli et al.: Multimode vs. single-mode interferometric phase, Online Material $p 8$

$$
\begin{aligned}
& <A_{r}^{2}>_{\Phi}=\frac{\int P_{1}(\boldsymbol{r}) P_{2}(\boldsymbol{r}+\lambda \boldsymbol{f}) P_{1}\left(\boldsymbol{r}^{\prime}\right) P_{2}\left(\boldsymbol{r}^{\prime}+\lambda \boldsymbol{f}^{\prime}\right)<\cos \left(\phi_{1}^{r}(\boldsymbol{r})-\phi_{2}^{r}(\boldsymbol{r}+\lambda \boldsymbol{f})\right) \cos \left(\phi_{1}^{r}\left(\boldsymbol{r}^{\prime}\right)-\phi_{2}^{r}\left(\boldsymbol{r}^{\prime}+\lambda \boldsymbol{f}^{\prime}\right)\right)>_{\Phi} \mathrm{d} \boldsymbol{r} \mathrm{d} \boldsymbol{r}^{\prime} \mathrm{d} \boldsymbol{f} \mathrm{d} \boldsymbol{f}^{\prime}}{\left[\int P_{1}(\boldsymbol{r}) \mathrm{d} \boldsymbol{r} \int P_{2}(\boldsymbol{r}) \mathrm{d} \boldsymbol{r}\right]^{2}} \\
& \text { using } \cos (a) \cos (b)=(\cos (a-b)+\cos (a+b)) / 2 \\
& =\frac{1}{2} \frac{\int P_{1}(\boldsymbol{r}) P_{2}(\boldsymbol{r}+\lambda \boldsymbol{f}) P_{1}\left(\boldsymbol{r}^{\prime}\right) P_{2}\left(\boldsymbol{r}^{\prime}+\lambda \boldsymbol{f}^{\prime}\right)<\cos \left(\phi_{1}^{r}(\boldsymbol{r})-\phi_{2}^{r}(\boldsymbol{r}+\lambda \boldsymbol{f})-\phi_{1}^{r}\left(\boldsymbol{r}^{\prime}\right)+\phi_{2}^{r}\left(\boldsymbol{r}^{\prime}+\lambda \boldsymbol{f}^{\prime}\right)>_{\Phi} \mathrm{d} \boldsymbol{r} \mathrm{d} \boldsymbol{r}^{\prime} \mathrm{d} \boldsymbol{f} \mathrm{d} \boldsymbol{f}^{\prime}\right.}{\left[\int P_{1}(\boldsymbol{r}) \mathrm{d} \boldsymbol{r} \int P_{2}(\boldsymbol{r}) \mathrm{d} \boldsymbol{r}\right]^{2}} \\
& +\frac{1}{2} \frac{\int P_{1}(\boldsymbol{r}) P_{2}(\boldsymbol{r}+\lambda \boldsymbol{f}) P_{1}\left(\boldsymbol{r}^{\prime}\right) P_{2}\left(\boldsymbol{r}^{\prime}+\lambda \boldsymbol{f}^{\prime}\right)<\cos \left(\phi_{1}^{r}(\boldsymbol{r})-\phi_{2}^{r}(\boldsymbol{r}+\lambda \boldsymbol{f})+\phi_{1}^{r}\left(\boldsymbol{r}^{\prime}\right)-\phi_{2}^{r}\left(\boldsymbol{r}^{\prime}+\lambda \boldsymbol{f}^{\prime}\right)\right)>_{\Phi} \mathrm{d} \boldsymbol{r} \mathrm{d} \boldsymbol{r}^{\prime} \mathrm{d} \boldsymbol{f} \mathrm{d} \boldsymbol{f}^{\prime}}{\left[\int P_{1}(\boldsymbol{r}) \mathrm{d} \boldsymbol{r} \int P_{2}(\boldsymbol{r}) \mathrm{d} \boldsymbol{r}\right]^{2}} \\
& \text { change of variables } \boldsymbol{r}+\lambda \boldsymbol{f} \rightarrow u, \boldsymbol{r}^{\prime}+\lambda \boldsymbol{f}^{\prime} \rightarrow \boldsymbol{u}^{\prime} \text { : } \\
& =\frac{1}{2} \frac{\int P_{1}(\boldsymbol{r}) P_{1}\left(\boldsymbol{r}^{\prime}\right) \exp ^{-\frac{1}{2} \mathcal{D}_{\phi_{r}}\left(\boldsymbol{r}, \boldsymbol{r}^{\prime}\right)} \mathrm{d} \boldsymbol{r} \mathrm{d} \boldsymbol{r}^{\prime} \int P_{2}(\boldsymbol{r}) P_{2}\left(\boldsymbol{r}^{\prime}\right) \exp ^{-\frac{1}{2} \mathcal{D}_{\phi_{r}}\left(\boldsymbol{r}, \boldsymbol{r}^{\prime}\right)} \mathrm{d} \boldsymbol{r} \mathrm{d} \boldsymbol{r}^{\prime}}{\left[\int P_{1}(\boldsymbol{r}) \mathrm{d} \boldsymbol{r} \int P_{2}(\boldsymbol{r}) \mathrm{d} \boldsymbol{r}\right]^{2}} \\
& +\mathrm{e}^{-4 \sigma_{\phi_{r}}^{2}} \frac{\int P_{1}(\boldsymbol{r}) P_{1}\left(\boldsymbol{r}^{\prime}\right) \exp ^{\frac{1}{2} \mathcal{D}_{\phi_{r}}\left(\boldsymbol{r}, \boldsymbol{r}^{\prime}\right)} \mathrm{d} \boldsymbol{r} \mathrm{d} \boldsymbol{r}^{\prime} \int P_{2}(\boldsymbol{r}) P_{2}\left(\boldsymbol{r}^{\prime}\right) \exp ^{\frac{1}{2} \mathcal{D}_{\phi_{r}}\left(\boldsymbol{r}, \boldsymbol{r}^{\prime}\right)} \mathrm{d} \boldsymbol{r} \mathrm{d} \boldsymbol{r}^{\prime}}{\left[\int P_{1}(\boldsymbol{r}) \mathrm{d} \boldsymbol{r} \int P_{2}(\boldsymbol{r}) \mathrm{d} \boldsymbol{r}\right]^{2}} \\
& <A_{i}^{2}>_{\Phi}=\frac{\int P_{1}(\boldsymbol{r}) P_{2}(\boldsymbol{r}+\lambda \boldsymbol{f}) P_{1}\left(\boldsymbol{r}^{\prime}\right) P_{2}\left(\boldsymbol{r}^{\prime}+\lambda \boldsymbol{f}^{\prime}\right)<\sin \left(\phi_{1}^{r}(\boldsymbol{r})-\phi_{2}^{r}(\boldsymbol{r}+\lambda \boldsymbol{f})\right) \sin \left(\phi_{1}^{r}\left(\boldsymbol{r}^{\prime}\right)-\phi_{2}^{r}\left(\boldsymbol{r}^{\prime}+\lambda \boldsymbol{f}^{\prime}\right)\right)>_{\Phi} \mathrm{d} \boldsymbol{r} \mathrm{d} \boldsymbol{r}^{\prime} \mathrm{d} \boldsymbol{f} \mathrm{d} \boldsymbol{f}^{\prime}}{\left[\int P_{1}(\boldsymbol{r}) \mathrm{d} \boldsymbol{r} \int P_{2}(\boldsymbol{r}) \mathrm{d} \boldsymbol{r}\right]^{2}} \\
& \text { using } \sin (a) \sin (b)=(\cos (a-b)-\cos (a+b)) / 2 \\
& =\frac{1}{2} \frac{\int P_{1}(\boldsymbol{r}) P_{2}(\boldsymbol{r}+\lambda \boldsymbol{f}) P_{1}\left(\boldsymbol{r}^{\prime}\right) P_{2}\left(\boldsymbol{r}^{\prime}+\lambda \boldsymbol{f}^{\prime}\right)<\cos \left(\phi_{1}^{r}(\boldsymbol{r})-\phi_{2}^{r}(\boldsymbol{r}+\lambda \boldsymbol{f})-\phi_{1}^{r}\left(\boldsymbol{r}^{\prime}\right)+\phi_{2}^{r}\left(\boldsymbol{r}^{\prime}+\lambda \boldsymbol{f}^{\prime}\right)>_{\Phi} \mathrm{d} \boldsymbol{r} \mathrm{d} \boldsymbol{r}^{\prime} \mathrm{d} \boldsymbol{f} \mathrm{d} \boldsymbol{f}^{\prime}\right.}{\left[\int P_{1}(\boldsymbol{r}) \mathrm{d} \boldsymbol{r} \int P_{2}(\boldsymbol{r}) \mathrm{d} \boldsymbol{r}\right]^{2}} \\
& -\frac{1}{2} \frac{\int P_{1}(\boldsymbol{r}) P_{2}(\boldsymbol{r}+\lambda \boldsymbol{f}) P_{1}\left(\boldsymbol{r}^{\prime}\right) P_{2}\left(\boldsymbol{r}^{\prime}+\lambda \boldsymbol{f}^{\prime}\right)<\cos \left(\phi_{1}^{r}(\boldsymbol{r})-\phi_{2}^{r}(\boldsymbol{r}+\lambda \boldsymbol{f})+\phi_{1}^{r}\left(\boldsymbol{r}^{\prime}\right)-\phi_{2}^{r}\left(\boldsymbol{r}^{\prime}+\lambda \boldsymbol{f}^{\prime}\right)>_{\Phi} \mathrm{d} \boldsymbol{r} \mathrm{d} \boldsymbol{r}^{\prime} \mathrm{d} \boldsymbol{f} \mathrm{d} \boldsymbol{f}^{\prime}\right.}{\left[\int P_{1}(\boldsymbol{r}) \mathrm{d} \boldsymbol{r} \int P_{2}(\boldsymbol{r}) \mathrm{d} \boldsymbol{r}\right]^{2}} \\
& \text { change of variables } \boldsymbol{r}+\lambda \boldsymbol{f} \rightarrow \boldsymbol{u}, \boldsymbol{r}^{\prime}+\lambda \boldsymbol{f}^{\prime} \rightarrow \boldsymbol{u}^{\prime} \text { : } \\
& =\frac{1}{2} \frac{\int P_{1}(\boldsymbol{r}) P_{1}\left(\boldsymbol{r}^{\prime}\right) \exp ^{-\frac{1}{2} \mathcal{D}_{\phi_{r}}\left(\boldsymbol{r}, \boldsymbol{r}^{\prime}\right)} \mathrm{d} \boldsymbol{r} \mathrm{d} \boldsymbol{r}^{\prime} \int P_{2}(\boldsymbol{r}) P_{2}\left(\boldsymbol{r}^{\prime}\right) \exp ^{-\frac{1}{2} \mathcal{D}_{\phi_{r}}\left(\boldsymbol{r}, \boldsymbol{r}^{\prime}\right)} \mathrm{d} \boldsymbol{r} \mathrm{d} \boldsymbol{r}^{\prime}}{\left[\int P_{1}(\boldsymbol{r}) \mathrm{d} \boldsymbol{r} \int P_{2}(\boldsymbol{r}) \mathrm{d} \boldsymbol{r}\right]^{2}} \\
& -\mathrm{e}^{-4 \sigma_{\phi_{r}}^{2}} \frac{\int P_{1}(\boldsymbol{r}) P_{1}\left(\boldsymbol{r}^{\prime}\right) \exp ^{\frac{1}{2} \mathcal{D}_{\phi_{r}}\left(\boldsymbol{r}, \boldsymbol{r}^{\prime}\right)} \mathrm{d} \boldsymbol{r} \mathrm{d} \boldsymbol{r}^{\prime} \int P_{2}(\boldsymbol{r}) P_{2}\left(\boldsymbol{r}^{\prime}\right) \exp ^{\frac{1}{2} \mathcal{D}_{\phi_{r}}\left(\boldsymbol{r}, \boldsymbol{r}^{\prime}\right)} \mathrm{d} \boldsymbol{r} \mathrm{d} \boldsymbol{r}^{\prime}}{\left[\int P_{1}(\boldsymbol{r}) \mathrm{d} \boldsymbol{r} \int P_{2}(\boldsymbol{r}) \mathrm{d} \boldsymbol{r}\right]^{2}}
\end{aligned}
$$

Putting Eqs. B.12, B.13, B.14 B.15 in Eqs. B.2. B.3, we obtain the theoretical expressions of the parameters $a$ and $\sigma$ of the Rician distribution is the single-mode case, as summarized in Table B.1 of the appendix.

\section{Appendix C: Astrometric phase error}

The astrometric phase error writes:

$$
\sigma_{\Delta_{\phi}^{\Delta \alpha h}}^{2}=2 \sigma_{\phi}^{2}-2 \operatorname{cov}\left(\phi_{s}, \phi_{r}^{\Delta \alpha h}\right)
$$

where $\phi_{s}$, and $\phi_{r}^{\Delta \alpha h}$ are respectively the partially AO-corrected phase of the astrophysical target and the phase estimated from the off-axis reference source. $\boldsymbol{\Delta} \boldsymbol{\alpha}$ is the angular separation between both sources, and h is the altitude of the turbulent layer.

Since $\sigma_{\phi}^{2}$ has been computed in previous sections, it remains to compute the covariance part of Eq. (C.1). The formal expression of the covariance is given by Chelli (1989):

$$
\operatorname{cov}\left(\phi_{s}, \phi_{r}^{\Delta \alpha h}\right)=\frac{1}{2} \frac{\operatorname{Re}\left[\mathrm{E}\left(Q Q_{\Delta \alpha h}^{*}\right)\right]-\operatorname{Re}\left[\mathrm{E}\left(Q Q_{\Delta \alpha h}\right)\right]}{\mathrm{E}(Q) \mathrm{E}\left(Q_{\Delta \alpha h}\right)}
$$

where $Q_{\Delta \alpha h}$ is the estimator of the coherent flux for the off-axis reference source.

\section{C.1. Multimode case}

Using formalism of Sect. A, one can derive the moments of the multimode estimator:

$$
\begin{aligned}
\mathrm{E}(Q) & =N \sqrt{t_{1} t_{2}} V_{12}<S_{12}\left(\boldsymbol{f}_{12}\right)>_{\phi} \\
\mathrm{E}\left(Q_{\Delta \alpha h}\right) & =N^{\Delta \alpha h} \sqrt{t_{1} t_{2}} V_{12}^{\Delta \alpha h}<S_{12}^{\Delta \alpha h}\left(\boldsymbol{f}_{12}\right)>_{\phi} \\
\mathrm{E}\left(Q Q_{\Delta \alpha h}^{*}\right) & =N N^{\Delta \alpha h} t_{1} t_{2} V_{12}\left(V_{12}^{\Delta \alpha h}\right)^{*}<S_{12}\left(\boldsymbol{f}_{12}\right)\left(S_{12}^{\Delta \alpha h}\right)^{*}\left(\boldsymbol{f}_{12}\right)>_{\phi} \\
\mathrm{E}\left(Q Q_{\Delta \alpha h}\right) & =N N^{\Delta \alpha h} t_{1} t_{2} V_{12} V_{12}^{\Delta \alpha h}<S_{12}\left(\boldsymbol{f}_{12}\right) S_{12}^{\Delta \alpha h}\left(\boldsymbol{f}_{12}\right)>_{\phi}
\end{aligned}
$$


E. Tatulli et al.: Multimode vs. single-mode interferometric phase, Online Material $p 9$

Table B.1. Parameters $a$ and $\sigma$ of the Rician probability distribution function for both multimode and single-mode cases.

Multimode case:
$a^{2}=\exp ^{-2 \sigma_{\phi_{r}}^{2}} \sqrt{\left(2-\exp ^{-4 \sigma_{\phi_{r}}^{2}}\left[\frac{\int\left[P(\boldsymbol{r}) P\left(\boldsymbol{r}^{\prime}\right) \mathrm{e}^{\left.\frac{1}{2} \mathcal{D}_{\phi_{r}}\left(\boldsymbol{r}, \boldsymbol{r}^{\prime}\right)\right]^{2} \mathrm{~d} \boldsymbol{r} \mathrm{d} \boldsymbol{r}^{\prime}}\right.}{\left[\int P^{2}(\boldsymbol{r}) \mathrm{d} \boldsymbol{r}\right]^{2}}\right)\right.}$
$\sigma^{2}=\frac{1}{2}\left(\frac{\int\left[P(\boldsymbol{r}) P\left(\boldsymbol{r}^{\prime}\right) \mathrm{e}^{\left.-\frac{1}{2} \mathcal{D}_{\phi_{r}}\left(\boldsymbol{r}, \boldsymbol{r}^{\prime}\right)\right]^{2}} \mathrm{~d} \boldsymbol{r} \mathrm{d} \boldsymbol{r}^{\prime}\right.}{\left[\int P^{2}(\boldsymbol{r}) \mathrm{d} \boldsymbol{r}\right]^{2}}-a^{2}\right)$

$$
\begin{aligned}
& \text { Single-mode case: } \\
& a^{2}=\exp ^{-2 \sigma_{\phi_{r}}^{2}} \sqrt{\left(2-\exp ^{-4 \sigma_{\phi_{r}}^{2}\left[\frac{\left[\int P(\boldsymbol{r}) P\left(\boldsymbol{r}^{\prime}\right) \mathrm{e}^{\frac{1}{2} \mathcal{D}_{\phi_{r}}\left(\boldsymbol{r}, \boldsymbol{r}^{\prime}\right)} \mathrm{d} \boldsymbol{r} \mathrm{d} \boldsymbol{r}^{\prime}\right]^{2}}{\left[\int P(\boldsymbol{r}) \mathrm{d} \boldsymbol{r}\right]^{4}}\right]^{2}}\right)} \\
& \sigma^{2}=\frac{1}{2}\left(\frac{\left[\int P(\boldsymbol{r}) P\left(\boldsymbol{r}^{\prime}\right) \mathrm{e}^{-\frac{1}{2} \mathcal{D}_{\phi_{r}}\left(\boldsymbol{r}, \boldsymbol{r}^{\prime}\right)} \mathrm{d} \boldsymbol{r} \mathrm{d} \boldsymbol{r}^{\prime}\right]^{2}}{\left[\int P(\boldsymbol{r}) \mathrm{d} \boldsymbol{r}\right]^{4}}-a^{2}\right)
\end{aligned}
$$

where $N^{\Delta \alpha h}$ and $V_{12}^{\Delta \alpha h}$ are respectively the number of photons (per surface unit and per time unit) and the visibility of the reference source, and with:

$<S_{12}^{\Delta \alpha h}\left(\boldsymbol{f}_{12}\right)>_{\Phi}=\int P_{1}(\boldsymbol{r}) P_{2}\left(\boldsymbol{r}+\lambda \boldsymbol{f}_{12}\right)<\exp ^{i\left(\phi_{1}^{\Delta \alpha h}(\boldsymbol{r})-\phi_{2}^{\Delta \alpha h}\left(\boldsymbol{r}+\lambda \boldsymbol{f}_{12}\right)\right)}>_{\Phi} \mathrm{d} \boldsymbol{r}=\Sigma_{P} \exp ^{-\sigma_{\phi_{r}}^{2}}$

Since we assume one turbulent layer located at the altitude $h$, we have by definition $\phi_{1}^{\Delta \alpha h}(\boldsymbol{r})=\phi_{1}(\boldsymbol{r}+\boldsymbol{\Delta} \boldsymbol{\alpha} h)$ and $\phi_{2}^{\Delta \alpha h}(\boldsymbol{r})=\phi_{2}(\boldsymbol{r}+\boldsymbol{\Delta} \boldsymbol{\alpha} h)$, hence:

$<S_{12}^{\Delta \alpha h}\left(\boldsymbol{f}_{12}\right)>_{\Phi}=\int P_{1}(\boldsymbol{r}) P_{2}\left(\boldsymbol{r}+\lambda \boldsymbol{f}_{12}\right)<\exp ^{i\left(\phi_{1}(\boldsymbol{r}+\boldsymbol{\Delta} \boldsymbol{\alpha} h)-\phi_{2}\left(\boldsymbol{r}+\lambda \boldsymbol{f}_{12}+\boldsymbol{\Delta} \boldsymbol{\alpha} h\right)\right)}>_{\Phi} \mathrm{d} \boldsymbol{r}=\Sigma_{P} \exp ^{-\sigma_{\phi_{r}}^{2}}$

Furthermore, we have:

$<S_{12}\left(\boldsymbol{f}_{12}\right) S_{12}^{\Delta \alpha h}\left(\boldsymbol{f}_{12}\right)>_{\Phi}$

$=\int P_{1}(\boldsymbol{r}) P_{2}\left(\boldsymbol{r}+\lambda \boldsymbol{f}_{12}\right) P_{1}\left(\boldsymbol{r}^{\prime}\right) P_{2}\left(\boldsymbol{r}^{\prime}+\lambda \boldsymbol{f}_{12}\right)<\exp ^{i\left(\phi_{1}(\boldsymbol{r})-\phi_{2}\left(\boldsymbol{r}+\lambda \boldsymbol{f}_{12}\right)+\phi_{1}\left(\boldsymbol{r}^{\prime}+\boldsymbol{\Delta} \boldsymbol{\alpha} h\right)-\phi_{2}\left(\boldsymbol{r}^{\prime}+\lambda \boldsymbol{f}_{12}+\boldsymbol{\Delta} \boldsymbol{\alpha} h\right)\right)}>_{\Phi} \mathrm{d} \boldsymbol{r} \mathrm{d} \boldsymbol{r}^{\prime}$

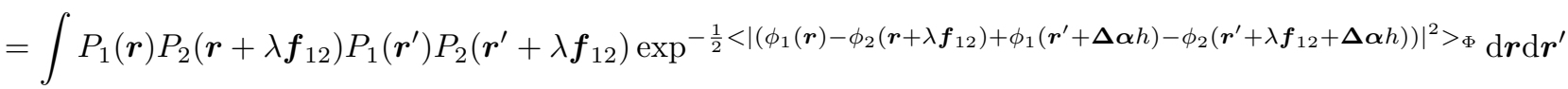

knowing that $<\phi_{1}(\boldsymbol{r}) \phi_{1}\left(\boldsymbol{r}^{\prime}+\boldsymbol{\Delta} \boldsymbol{\alpha} h\right)>_{\Phi}=\sigma_{\phi_{r}}^{2}-\frac{1}{2} \mathcal{D}_{\phi_{r}}\left(\boldsymbol{r}, \boldsymbol{r}^{\prime}+\boldsymbol{\Delta} \boldsymbol{\alpha} h\right)$, it comes,

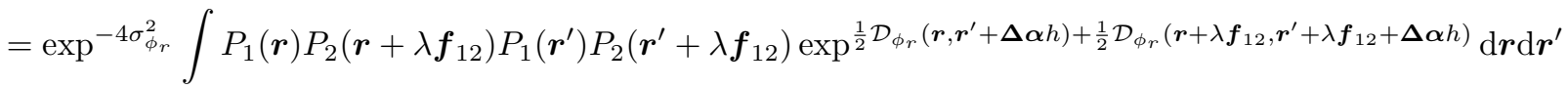
changing axes reference: $P_{1}, P_{2}$ centered on 0 ,

$=\exp ^{-4 \sigma_{\phi_{r}}^{2}} \int\left[P(\boldsymbol{r}) P\left(\boldsymbol{r}^{\prime}\right) \exp ^{\frac{1}{2} \mathcal{D}_{\phi_{r}}\left(\boldsymbol{r}, \boldsymbol{r}^{\prime}+\boldsymbol{\Delta} \boldsymbol{\alpha} h\right)}\right]\left[P(\boldsymbol{r}) P\left(\boldsymbol{r}^{\prime}\right) \exp ^{\frac{1}{2} \mathcal{D}_{\phi_{r}}\left(\boldsymbol{r}, \boldsymbol{r}^{\prime}+\boldsymbol{\Delta} \boldsymbol{\alpha} h\right)}\right] \mathrm{d} \boldsymbol{r} \mathrm{d} \boldsymbol{r}^{\prime}$

$<S_{12}\left(\boldsymbol{f}_{12}\right)\left(S_{12}^{\Delta \alpha h}\right)^{*}\left(\boldsymbol{f}_{12}\right)>_{\Phi}$

$=\int P_{1}(\boldsymbol{r}) P_{2}\left(\boldsymbol{r}+\lambda \boldsymbol{f}_{12}\right) P_{1}\left(\boldsymbol{r}^{\prime}\right) P_{2}\left(\boldsymbol{r}^{\prime}+\lambda \boldsymbol{f}_{12}\right)<\exp ^{i\left(\phi_{1}(\boldsymbol{r})-\phi_{2}\left(\boldsymbol{r}+\lambda \boldsymbol{f}_{12}\right)-\phi_{1}\left(\boldsymbol{r}^{\prime}+\boldsymbol{\Delta} \boldsymbol{\alpha} h\right)+\phi_{2}\left(\boldsymbol{r}^{\prime}+\lambda \boldsymbol{f}_{12}+\Delta \boldsymbol{\alpha} h\right)\right)}>_{\Phi} \mathrm{d} \boldsymbol{r} \mathrm{d} \boldsymbol{r}^{\prime}$

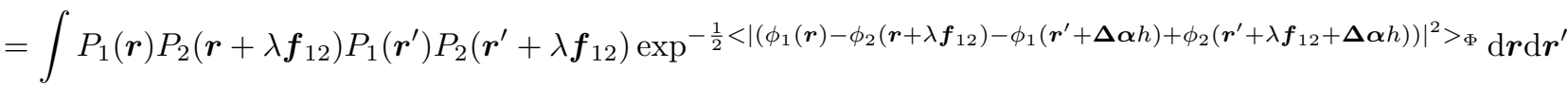

$=\int P_{1}(\boldsymbol{r}) P_{2}\left(\boldsymbol{r}+\lambda \boldsymbol{f}_{12}\right) P_{1}\left(\boldsymbol{r}^{\prime}\right) P_{2}\left(\boldsymbol{r}^{\prime}+\lambda \boldsymbol{f}_{12}\right) \exp ^{-\frac{1}{2} \mathcal{D}_{\phi_{r}}\left(\boldsymbol{r}, \boldsymbol{r}^{\prime}+\boldsymbol{\Delta} \boldsymbol{\alpha} h\right)-\frac{1}{2} \mathcal{D}_{\phi_{r}}\left(\boldsymbol{r}+\lambda \boldsymbol{f}_{12}, \boldsymbol{r}^{\prime}+\lambda \boldsymbol{f}_{12}+\boldsymbol{\Delta} \boldsymbol{\alpha} h\right)} \mathrm{d} \boldsymbol{r} \mathrm{d} \boldsymbol{r}^{\prime}$

changing axes reference: $P_{1}, P_{2}$ centered on 0 ,

$\left.=\int\left[P(\boldsymbol{r}) P\left(\boldsymbol{r}^{\prime}\right)\right) \exp ^{-\frac{1}{2} \mathcal{D}_{\phi_{r}}\left(\boldsymbol{r}, \boldsymbol{r}^{\prime}+\boldsymbol{\Delta} \boldsymbol{\alpha} h\right)}\right]\left[P\left(\boldsymbol{r} P\left(\boldsymbol{r}^{\prime}\right) \exp ^{-\frac{1}{2} \mathcal{D}_{\phi_{r}}\left(\boldsymbol{r}, \boldsymbol{r}^{\prime}+\boldsymbol{\Delta} \boldsymbol{\alpha} h\right)}\right] \mathrm{d} \boldsymbol{r} \mathrm{d} \boldsymbol{r}^{\prime}\right.$ 


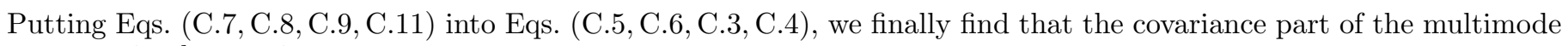
astrometric phase writes:

$$
\operatorname{cov}\left(\phi_{s}, \phi_{r}^{\Delta \alpha h}\right)=\frac{1}{2} \frac{\int\left[P(\boldsymbol{r}) P\left(\boldsymbol{r}^{\prime}\right) \mathrm{e}^{-\frac{1}{2} \mathcal{D}_{\phi_{r}}\left(\boldsymbol{r}, \boldsymbol{r}^{\prime}+\boldsymbol{\Delta} \boldsymbol{\alpha} h\right)}\right]^{2} \mathrm{~d} \boldsymbol{r} \mathrm{d} \boldsymbol{r}^{\prime}-\mathrm{e}^{-4 \sigma_{\phi_{r}}^{2} \int\left[P(\boldsymbol{r}) P\left(\boldsymbol{r}^{\prime}\right) \mathrm{e}^{\frac{1}{2} \mathcal{D}_{\phi_{r}}\left(\boldsymbol{r}, \boldsymbol{r}^{\prime}+\boldsymbol{\Delta} \boldsymbol{\alpha} h\right)}\right]^{2} \mathrm{~d} \boldsymbol{r} \mathrm{d} \boldsymbol{r}^{\prime}}}{\left[\int P^{2}(\boldsymbol{r}) \mathrm{d} \boldsymbol{r}\right]^{2} \mathrm{e}^{-2 \sigma_{\phi_{r}}^{2}}}
$$

\section{C.2. Single-mode case}

We once again use formalism of Sect. A to compute the moments of the single-mode estimator:

$$
\begin{aligned}
\mathrm{E}(Q) & =\Sigma_{P} N \sqrt{t_{1} t_{2}} \rho_{0} V_{12}<\rho_{12}>_{\phi} \\
\mathrm{E}\left(Q_{\Delta \alpha h}\right) & =\Sigma_{P} N^{\Delta \alpha h} \sqrt{t_{1} t_{2}} \rho_{0} V_{12}^{\Delta \alpha h}<\rho_{12}^{\Delta \alpha h}>_{\phi} \\
\mathrm{E}\left(Q Q_{\Delta \alpha h}^{*}\right) & =\Sigma_{P}^{2} N N^{\Delta \alpha h} t_{1} t_{2} \rho_{0}^{2} V_{12}\left(V_{12}^{\Delta \alpha h}\right)^{*}<\rho_{12}\left(\rho_{12}^{\Delta \alpha h}\right)^{*}>_{\phi} \\
\mathrm{E}\left(Q Q_{\Delta \alpha h}\right) & =\Sigma_{P}^{2} N N^{\Delta \alpha h} t_{1} t_{2} \rho_{0}^{2} V_{12} V_{12}^{\Delta \alpha h}<\rho_{12} \rho_{12}^{\Delta \alpha h}>_{\phi}
\end{aligned}
$$

with

$$
\begin{aligned}
<\rho_{12}>_{\Phi} & =\mathrm{e}^{-\sigma_{\phi_{r}}^{2}} \\
<\rho_{12}^{\Delta \alpha h}>_{\Phi} & =\mathrm{e}^{-\sigma_{\phi_{r}}^{2}}
\end{aligned}
$$

We also have:

$$
\begin{aligned}
& <\rho_{12} \rho_{12}^{\Delta \alpha h}>_{\Phi}=\frac{P_{1}(\boldsymbol{r}) P_{2}(\boldsymbol{r}+\lambda \boldsymbol{f}) P_{1}\left(\boldsymbol{r}^{\prime}\right) P_{2}\left(\boldsymbol{r}^{\prime}+\lambda \boldsymbol{f}^{\prime}\right)<\exp ^{i\left(\phi_{1}(\boldsymbol{r})+\phi_{1}\left(\boldsymbol{r}^{\prime}+\Delta \boldsymbol{\alpha} h\right)-\phi_{2}(\boldsymbol{r}+\lambda \boldsymbol{f})-\phi_{2}\left(\boldsymbol{r}^{\prime}+\lambda \boldsymbol{f}^{\prime}+\Delta \boldsymbol{\alpha} h\right)\right)}>_{\Phi} \mathrm{d} \boldsymbol{r} \mathrm{d} \boldsymbol{r}^{\prime} \mathrm{d} \boldsymbol{f} \mathrm{d} \boldsymbol{f}^{\prime}}{\left[\int P_{1}(\boldsymbol{r}) \mathrm{d} \boldsymbol{r} \int P_{2}(\boldsymbol{r}) \mathrm{d} \boldsymbol{r}\right]^{2}} \\
& =\mathrm{e}^{-4 \sigma_{\phi_{r}}^{2}} \frac{\int P_{1}(\boldsymbol{r}) P_{2}(\boldsymbol{r}+\lambda \boldsymbol{f}) P_{1}\left(\boldsymbol{r}^{\prime}\right) P_{2}\left(\boldsymbol{r}^{\prime}+\lambda \boldsymbol{f}^{\prime}\right) \exp ^{\frac{1}{2} \mathcal{D}_{\phi_{r}}\left(\boldsymbol{r}, \boldsymbol{r}^{\prime}+\boldsymbol{\Delta} \boldsymbol{\alpha} h\right)+\frac{1}{2} \mathcal{D}_{\phi_{r}}\left(\boldsymbol{r}+\lambda \boldsymbol{f}, \boldsymbol{r}^{\prime}+\lambda \boldsymbol{f}^{\prime}+\boldsymbol{\Delta} \boldsymbol{\alpha} h\right)} \mathrm{d} \boldsymbol{r} \mathrm{d} \boldsymbol{r}^{\prime} \mathrm{d} \boldsymbol{f} \mathrm{d} \boldsymbol{f}^{\prime}}{\left[\int P_{1}(\boldsymbol{r}) \mathrm{d} \boldsymbol{r} \int P_{2}(\boldsymbol{r}) \mathrm{d} \boldsymbol{r}\right]^{2}} \\
& \text { change of variables } \boldsymbol{r}+\lambda \boldsymbol{f} \rightarrow \boldsymbol{u}, \boldsymbol{r}^{\prime}+\lambda \boldsymbol{f}^{\prime} \rightarrow \boldsymbol{u}^{\prime} \text { : }
\end{aligned}
$$

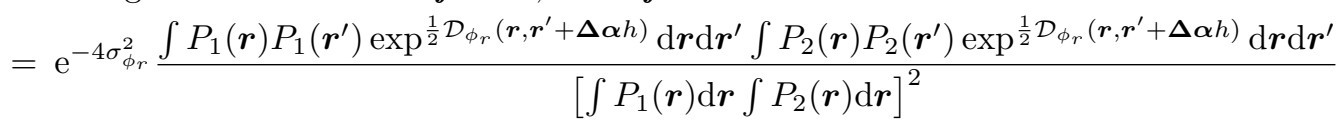

$$
\begin{aligned}
& <\rho_{12}\left(\rho_{12}^{\Delta \boldsymbol{\alpha} h}\right)^{*}>_{\Phi}=\frac{P_{1}(\boldsymbol{r}) P_{2}(\boldsymbol{r}+\lambda \boldsymbol{f}) P_{1}\left(\boldsymbol{r}^{\prime}\right) P_{2}\left(\boldsymbol{r}^{\prime}+\lambda \boldsymbol{f}^{\prime}\right)<\exp ^{i\left(\phi_{1}(\boldsymbol{r})-\phi_{1}\left(\boldsymbol{r}^{\prime}+\Delta \boldsymbol{\alpha} h\right)-\phi_{2}(\boldsymbol{r}+\lambda \boldsymbol{f})+\phi_{2}\left(\boldsymbol{r}^{\prime}+\lambda \boldsymbol{f}^{\prime}+\Delta \boldsymbol{\alpha} h\right)\right)}>_{\Phi} \mathrm{d} \boldsymbol{r} \mathrm{d} \boldsymbol{r}^{\prime} \mathrm{d} \boldsymbol{f} \mathrm{d} \boldsymbol{f}^{\prime}}{\left[\int P_{1}(\boldsymbol{r}) \mathrm{d} \boldsymbol{r} \int P_{2}(\boldsymbol{r}) \mathrm{d} \boldsymbol{r}\right]^{2}} \\
& =\frac{\int P_{1}(\boldsymbol{r}) P_{2}(\boldsymbol{r}+\lambda \boldsymbol{f}) P_{1}\left(\boldsymbol{r}^{\prime}\right) P_{2}\left(\boldsymbol{r}^{\prime}+\lambda \boldsymbol{f}^{\prime}\right) \exp ^{-\frac{1}{2} \mathcal{D}_{\phi_{r}}\left(\boldsymbol{r}, \boldsymbol{r}^{\prime}+\Delta \boldsymbol{\alpha} h\right)-\frac{1}{2} \mathcal{D}_{\phi_{r}}\left(\boldsymbol{r}+\lambda \boldsymbol{f}, \boldsymbol{r}^{\prime}+\lambda \boldsymbol{f}^{\prime}+\boldsymbol{\Delta} \boldsymbol{\alpha} h\right)} \mathrm{d} \boldsymbol{r} \mathrm{d} \boldsymbol{r}^{\prime} \mathrm{d} \boldsymbol{f} \mathrm{d} \boldsymbol{f}^{\prime}}{\left[\int P_{1}(\boldsymbol{r}) \mathrm{d} \boldsymbol{r} \int P_{2}(\boldsymbol{r}) \mathrm{d} \boldsymbol{r}\right]^{2}} \\
& \text { change of variables } \boldsymbol{r}+\lambda \boldsymbol{f} \rightarrow \boldsymbol{u}, \boldsymbol{r}^{\prime}+\lambda \boldsymbol{f}^{\prime} \rightarrow \boldsymbol{u}^{\prime} \text { : } \\
& =\frac{\int P_{1}(\boldsymbol{r}) P_{1}\left(\boldsymbol{r}^{\prime}\right) \exp ^{-\frac{1}{2} \mathcal{D}_{\phi_{r}}\left(\boldsymbol{r}, \boldsymbol{r}^{\prime}+\Delta \boldsymbol{\alpha} h\right)} \mathrm{d} \boldsymbol{r} \mathrm{d} \boldsymbol{r}^{\prime} \int P_{2}(\boldsymbol{r}) P_{2}\left(\boldsymbol{r}^{\prime}\right) \exp ^{-\frac{1}{2} \mathcal{D}_{\phi_{r}}\left(\boldsymbol{r}, \boldsymbol{r}^{\prime}+\boldsymbol{\Delta} \boldsymbol{\alpha} h\right)} \mathrm{d} \boldsymbol{r} \mathrm{d} \boldsymbol{r}^{\prime}}{\left[\int P_{1}(\boldsymbol{r}) \mathrm{d} \boldsymbol{r} \int P_{2}(\boldsymbol{r}) \mathrm{d} \boldsymbol{r}\right]^{2}}
\end{aligned}
$$

Putting Eqs. C.17, C.18, C.19 C.20 into Eqs. C.15, C.16, C.13 C.14, we find that the covariance part of the single-mode astrometric phase writes:

$$
\operatorname{cov}\left(\phi_{s}, \phi_{r}^{\Delta \alpha h}\right)=\frac{1}{2} \frac{\left[\int P(\boldsymbol{r}) P\left(\boldsymbol{r}^{\prime}\right) \mathrm{e}^{-\frac{1}{2} \mathcal{D}_{\phi_{r}}\left(\boldsymbol{r}, \boldsymbol{r}^{\prime}+\Delta \boldsymbol{\alpha} h\right)} \mathrm{d} \boldsymbol{r} \mathrm{d} \boldsymbol{r}^{\prime}\right]^{2}-\mathrm{e}^{-4 \sigma_{\phi_{r}}^{2}\left[\int P(\boldsymbol{r}) P\left(\boldsymbol{r}^{\prime}\right) \mathrm{e}^{\frac{1}{2} \mathcal{D}_{\phi_{r}}\left(\boldsymbol{r}, \boldsymbol{r}^{\prime}+\Delta \boldsymbol{\alpha} h\right)} \mathrm{d} \boldsymbol{r} \mathrm{d} \boldsymbol{r}^{\prime}\right]^{2}}}{\left[\int P(\boldsymbol{r}) \mathrm{d} \boldsymbol{r}\right]^{4} \mathrm{e}^{-2 \sigma_{\phi_{r}}^{2}}}
$$

\section{Appendix D: Phase spatial fluctuations in partial AO correction}

The spatial fluctuations of the atmospheric phase can be characterized by the computation of the so-called spatial structure function $\mathcal{D}_{\phi}\left(\boldsymbol{r}, \boldsymbol{r}^{\prime}\right)$ defined as the variance of the phase difference taken at two radii $\boldsymbol{r}$ and $\boldsymbol{r}^{\prime}$ :

$$
\mathcal{D}_{\phi}\left(\boldsymbol{r}, \boldsymbol{r}^{\prime}\right)=\mathcal{D}_{\phi}\left(\boldsymbol{r}-\boldsymbol{r}^{\prime}\right)=<\left|\phi(\boldsymbol{r})-\phi\left(\boldsymbol{r}^{\prime}\right)\right|^{2}>
$$

In the fully turbulent case, and assuming Kolmogorov's description of the atmosphere, the phase structure function can be written (Roddier 1979):

$$
\mathcal{D}_{\phi}(\boldsymbol{\rho})=6.88\left(\frac{\boldsymbol{\rho}}{r_{0}}\right)^{5 / 3}
$$


$r_{0}$ being the fried parameter, that gives the ultimate spatial resolution achievable in presence of atmospheric turbulence, that is $\theta_{\text {turb }}=\lambda / r_{0}$.

When partial AO correction is applied, low (spatial) orders of the corrugated wavefront are real-time compensated thanks to a deformable mirror. The variance $\sigma_{\phi_{r}}^{2}$ of the residual turbulent phase $\phi^{r}(\boldsymbol{r})$ not corrected by the system, can be approximated as following, providing a full correction of the first $J \ll 1$ Zernike modes of the turbulence Noll (1976):

$$
\sigma_{\phi_{r}}^{2} \simeq 0.2944 J^{-\sqrt{3 / 2}}\left(\frac{D}{r_{0}}\right)^{5 / 3}
$$

Furthermore, the phase structure function of the residual phase can be written:

$$
\mathcal{D}_{\phi_{r}}(\boldsymbol{\rho})=2 \sigma_{\phi_{r}}^{2}\left[1-\frac{<\phi^{r}(\boldsymbol{r})-\phi^{r}(\boldsymbol{r}+\boldsymbol{\rho})>}{\sigma_{\phi_{r}}^{2}}\right]
$$

For $\rho$ bigger than typically the distance between the mirror's actuators, the phases are uncorrelated, that is $<\phi^{r}(\boldsymbol{r})-$ $\phi^{r}(\boldsymbol{r}+\rho)>=0$, and consequently $\mathcal{D}_{\phi_{r}}$ saturates at $2 \sigma_{\phi_{r}}^{2}$.

The long exposure AO corrected transfer function of the atmosphere is by definition:

$$
B(\boldsymbol{f})=\exp \left[-\frac{1}{2} \mathcal{D}_{\phi_{r}}(\lambda \boldsymbol{f})\right]
$$

Taking into account the properties of the phase structure function of the residual phase, it comes (Conan 1994) that $B(\boldsymbol{f})$ can be decomposed in two parts: one low frequency term - namely the halo $\left(F T O_{\text {halo }}\right)$ - and one high frequency term saturating at $e^{-\sigma_{\phi_{r}}^{2}}$, namely the coherent energy. It comes that the long exposure transfer function of the telescope $T(\boldsymbol{f})=T_{0}(\boldsymbol{f}) \cdot B(\boldsymbol{f})$, where $T_{0}(\boldsymbol{f})$ is the transfer function of a perfect telescope, can be in first approximation modelled by a sum of two components:

$$
\begin{aligned}
T(f) & =T_{0}(f) \cdot\left[e^{-\sigma_{\phi_{r}}^{2}}+F T O_{\text {halo }}(f)\right] \\
& \simeq T_{0}(f) \cdot\left[e^{-\sigma_{\phi_{r}}^{2}}+\left(1-\exp ^{-\sigma_{\phi_{r}}^{2}}\right) \exp ^{-\frac{1}{2} \mathcal{D}_{\phi}(\lambda \boldsymbol{f})}\right]
\end{aligned}
$$

Eq. (D.4) also demonstrates that for interferometers equipped with AO systems, the phases $\phi_{1}^{r}$ and $\phi_{2}^{r}$ of telescopes 1 and 2 are always uncorrelated. As a consequence, the phase structure function of the residual piston phase $\phi_{12}=\phi_{1}^{r}-\phi_{2}^{r}$ is twice the phase structure function of the residual phase over one single telescope (assuming equivalent AO systems for both telescopes), that is $\mathcal{D}_{\phi_{12}}(\boldsymbol{\rho})=2 \mathcal{D}_{\phi_{r}}(\boldsymbol{\rho})$. As a matter of fact, even if no AO correction is applied, the phases $\phi_{1}$ and $\phi_{2}$ can still be consider uncorrelated, as soon as the baseline is longer than the outer scale of the atmosphere $\mathcal{L}_{0}$. This is for example the case for the VLTI at Paranal, where the outer scale has been estimated to be $\mathcal{L}_{0} \simeq 22 \mathrm{~m}($ Martin et al. 2000). 